\title{
Two-parameter model for optimizing target beam distribution with an octupole magnet
}

\author{
Shin-ichiro Meigo $\odot$ and Motoki Ooi \\ J-PARC Center, Japan Atomic Energy Agency, Tokai, Ibaraki 319-1195, Japan \\ Hiroshi Fujimori \\ J-PARC Center, High Energy Accelerator Research Organization (KEK), \\ Tsukuba, Ibaraki 305-0801, Japan
}

(Received 24 March 2020; accepted 3 June 2020; published 23 June 2020)

\begin{abstract}
As hadron accelerators achieve increasing beam power, damage to targets is becoming increasingly severe. To mitigate this damage, nonlinear beam optics based on octupole magnets is attractive. Nonlinear optics can decrease the beam-focusing hazard due to failure of the rastering magnet. As a side effect of nonlinear optics, the beam size at the tail is known to expand drastically compared with linear optics, owing to defocusing in the nonlinear case; this may cause severe beam loss downstream of the octupole magnets. Beam shape and beam loss as a side effect of nonlinear optics have thus far been studied via a simplified filament model that ignores beam-divergence spread at the octupole magnet, so that the problem may be treated by a simplified transfer matrix. Several beam-tracking studies have been performed for the specified case given by the specified emittance and Twiss parameters, whereas a simplified model is required for application to the pragmatic beam tuning. In this study, a new generalized model is proposed for application to an octupole magnet, regardless of the filament-model approximation. It is found that the transverse distribution obtained by beam tracking can be specified by the introduction of only two parameters, namely the normalized octupole strength of $K_{8}^{*}$ and the $\cot \phi$ of the phase advance from the octupole to the arbitrary position. With the new generalized model, the distribution with allowable beam loss is studied in detail. The best uniform shape is found for specific parameters of $K_{8}^{*}$ and $\cot \phi$ that cannot be deduced from the filament model. However, to achieve the flat distribution, a considerable $K_{8}^{*}$ is required, incurring a large beam loss at the position having $\cot \phi \sim-0.5$ downstream of the octupole. To achieve the two antagonistic requirements of reduction of the beam-peak density and minimization of the beam loss, the transverse distribution is surveyed for a large range of beam position. It is found that a bell-shaped distribution with $K_{8}^{*} \sim 1$ and $\cot \phi \sim 3$ can satisfy requirements. This result is applied to beam transport in the spallation neutron source at J-PARC. The beam profile calculated using nonlinear optics is compared with the experimental result. It is proven that the transverse beam distribution with nonlinear optics and an octupole magnet can be simplified by the present generalized model, which can be applied to other facilities using octupole magnets. The calculation result shows good agreement with the experimental data, and the peak current density is reduced by $50 \%$ compared with the linear-optics case with allowable beamwidth and beam loss.
\end{abstract}

DOI: 10.1103/PhysRevAccelBeams.23.062802

\section{INTRODUCTION}

With recent progress in the accelerator field, highintensity accelerators are now being planned for several applications. The advanced use is as an accelerator-driven system (ADS) to mitigate the hazard of long-lived actinides

Published by the American Physical Society under the terms of the Creative Commons Attribution 4.0 International license. Further distribution of this work must maintain attribution to the author(s) and the published article's title, journal citation, and DOI. such as ${ }^{237} \mathrm{~Np}$ and ${ }^{241} \mathrm{Am}$ produced in a nuclear reactor. An ADS consists of a subcritical core of the nuclear reactor and spallation neutron source driven by the high-intensity accelerator. The Japan Atomic Energy Agency (JAEA) [1] and multipurpose hybrid research reactor for high-tech applications (MYRRHA) [2] proposed by the Belgian nuclear research center (SCK - CEN) have a plan to develop an ADS using high-intensity proton accelerators with beam powers of 30 and $2.4 \mathrm{MW}$, respectively. In the practical aspect of the ADS, damage to the beam window is one of the critical issues.

Damage to the beam window is a major practical concern for an ADS. To mitigate such damage, it is necessary to 
decrease the peak current density at the window. Beam scanning or rastering is commonly used to decrease the time-averaged peak current density at many facilities for beam dumping and medical applications, while conserving the integrated beam current. To obtain a flat beam profile, beam rastering with pulse-kicker magnets to create a flat time-averaged profile is generally employed [3-5]. Typically, except for liquid-metal targets with short pulses, the damage to the target material is determined by the target-material displacement per atom, which is proportional to the peak density.

To develop beam-interception materials such as windows and targets utilized in high-intensity proton accelerators, the radiation damage in accelerator target environments (RaDIATE) [6] collaboration was established. This collaboration examined many materials for beam interception under irradiation by the $180-\mathrm{MeV}$ proton beam at the Brookhaven Linac Isotope Producer (BLIP) in Brookhaven National Laboratory (BNL). At BLIP, a round-shaped rastering was employed to give uniform damage to the samples. It was reported that the sample was entirely collapsed [6]. One reason for this is thought to be a failure of the rastering system.

A beam-rastering system is also planned for application at the European Spallation Neutron Source (ESS) [7], a new facility under construction with a 5-MW proton accelerator. As a countermeasure against the failure of rastering at ESS, a fast failure-detection system will be installed to stop the beam immediately. Due to high-level safety requirements for the ADS, countermeasures against rastering failure are even more important than at ESS, such that JAEA's ADS plan [1] does not consider rastering. To mitigate damage to the beam window, manipulation using nonlinear beam optics based on dc multipole magnets is preferable.

\section{A. Demands of nonlinear beam shaping for short pulse in high-intensity accelerator facilities}

The Japan Proton Accelerator Research Complex (J-PARC) [8] now houses a MW-class pulsed neutron source in the Materials and Life Science Experimental Facility (MLF) within the Japan Spallation Neutron Source (JSNS) [9] and the Muon Science Facility [10]. Since 2008, this source has produced a high-power proton beam of $500 \mathrm{~kW}$. To produce neutrons, a $3-\mathrm{GeV}$ proton beam collides with a mercury target; and to produce muons, the beam collides with a 2-cm-thick carbon graphite target. To use the proton beam for efficient particle production, both targets are aligned in a cascade scheme, with the graphite target placed $33 \mathrm{~m}$ upstream of the neutron target. Between the two targets, $8 \%$ beam loss is expected due to scattering at the carbon target. This amount of beam loss for 1-MW operation at JSNS has a beam power equivalent to half of the ISIS Neutron and Muon Source at the Rutherford Appleton Laboratory, which was the highestintensity pulse neutron source before JSNS and the
Spallation Neutron Source (SNS) at Oak Ridge National Laboratory (ORNL) [11] were established. Therefore, all components between two targets such as magnets can be exchanged by remote handling. For both sources, the $3-\mathrm{GeV}$ proton beam with a repetition rate of $25 \mathrm{~Hz}$ is delivered from a rapid cycling synchrotron (RCS) to the targets by the 3-GeV RCS to Neutron facility Beam Transport (3NBT) [12-14]. Before injection into the $\mathrm{RCS}$, the proton beam is accelerated up to $0.4 \mathrm{GeV}$ by a linac. The beam is then accumulated in two short bunches and accelerated up to $3 \mathrm{GeV}$ in the RCS. The extracted $3-\mathrm{GeV}$ proton beam, with a bunch width of $150 \mathrm{~ns}$ at full width at half maximum double bunched with a spacing of $600 \mathrm{~ns}$, is transferred to the muon-production target and the SNS.

Rastering can decrease only the first order of density dependence. In the case of a short-duration beam impinging on a liquid-metal target, scanning or rastering the beam with a deflecting magnetic field will not mitigate the pitting damage caused by the beam because this damage is proportional to the fourth power of the peak density. It is well known that the morphology of pitting-cavitation damage can be classified into roughly two stages: an incubation period and a steady-state period. The former is the early stage of cavitation damage, where plastic deformation of the surface is dominant without noticeable mass loss. The latter is the erosion stage, with significant mass loss occurring within a short period. It is reported that the incubation period is well described as the inverse time of the fourth power of the peak current density $[15,16]$. After incubation, serious damage is inflicted in the steadystate period. The incubation time is more important than the period afterwards. However, once the threshold is exceeded, erosion is expected to follow power-law dependence. Therefore, cavitation erosion of the target-vessel material is a significant concern in high-power operation of the JSNS and SNS facilities, and is predicted to be the life-limiting factor of the mercury target vessel.

Recently, pitting erosion became evident in the mercury target container [17-19], and the extent of the damage was proportional to the fourth power of the peak current density of the proton beam [20]. After operating the beam at high power, significant pitting erosion of the mercury target caused by pressure waves was observed in a spent target vessel at JSNS and at the SNS in the ORNL $[11,15]$. Using linear optics (i.e., quadrupole magnets) for beam transport, the peak current density can be reduced by expanding the beam at the target. However, beam expansion increases heat near the target, where the shielding, neutron reflector, and moderator are located. Therefore, the peak current density is limited by the heat induced near the target, the allowable limit of which is possibly lower than $1 \mathrm{~W} / \mathrm{cc}$. At the JSNS, the minimum peak current density is expected to be $9 \mu \mathrm{A} / \mathrm{cm}^{2}$, resulting in a thermal-energy density of $14 \mathrm{~J} / \mathrm{cc} /$ pulse at the target $[12,21]$. Thus far, mercury at 
the SNS has leaked octave from the target vessel, owing to pitting erosion under beam powers of 1 and $1.4 \mathrm{MW}$. To mitigate this erosion, small-helium-bubble-injection system was implemented at JSNS. The tiny helium bubbles capture the negative pressure produced in the mercury, which may cause pitting erosion of the vessel $[16,22]$. Although the vibration of the target vessel decreased, mitigation of the pitting damage is quantitatively unclear. Owing to the low repetition rate of $25 \mathrm{~Hz}$ at JSNS, which is $60 \mathrm{~Hz}$ in the case of SNS, the beam power of one shot and the peak heat density in mercury at the JSNS for a 1-MW beam is about twice as high as that in the present 1.4-MW SNS beam. Therefore, to mitigate the pitting erosion, a beam-flattening system for peak-density reduction is urgently required at JSNS.

Since damage is proportional to the fourth power of peak density before rastering, it remains proportional to the third power of peak current density using the rastering, which is insufficient to mitigate the pitting erosion. It should be noted that in the case of JSNS, the pulse length is shorter than $1 \mu \mathrm{s}$, so by rastering, a flat shape for each beam pulse is impossible to achieve because the pulse-kicker magnet does not have the required fast response time. Therefore, beam rastering cannot sufficiently mitigate pitting damage to the mercury target vessel. A beam-flattening system for each pulse is required for the mercury-target system, especially at JSNS. Accordingly, to reduce the peak current density, a beam-flattening system based on nonlinear beam optics with octupole magnets has been developed at JSNS.

\section{B. Previous studies of nonlinear optics for transverse beam manipulation}

In Ref. [23], transverse beam uniformization by the nonlinear-focusing method is studied theoretically. The developed formulas predict both the strength of the different nonlinear magnetic fields required for beam uniformization and the resultant width of the uniform region. The idea of beam uniformization was proposed by Meads Jr. in 1983 [24]. Kashy and Sherrill numerically demonstrated the formation of a uniform distribution using an octupole field considering the specific parameters of the beam line [25]. They verified that odd-order multipole fields such as octupole and dodecapole fields are needed for uniformization of a beam with a Gaussian profile [26]. Extensive practical studies were carried out in the 1990s. Blind numerically studied a method for size tuning and the effect of beam jitter in the case of a large-area target [27]. Batygin analytically calculated the nonlinear force required to produce a uniform beam in a simple beam line composed of a multipole lens and a drift space [28]. Méot and Aniel derived the octupole- and dodecapole-field strengths considering realistic beam optics $[29,30]$. Starting in the 1990s, experimental approaches were introduced as well. The first uniform-beam-formation experiment using octupole magnets was performed successfully at BNL [31,32]. In recent years, beam uniformization by means of this nonlinear focusing method has received considerable attention as an alternative uniform-irradiation method [33-41]. All previous results are focused on uniformity, but beam loss due to nonlinear optics has rarely been studied. To apply highintensity accelerators, it is essential to study both flatness and beam loss without the filament model.

In this paper, a detailed study based on the filamentapproximation model of nonlinear beam optics for a flattening beam system is described in Sec. II. In Sec. III, a new general model of nonlinear optics that can be applied in all cases without the filament approximation is proposed. The application of this system to JSNS and a comparison between the results of particle-tracking simulations and experiments are discussed in Sec. IV.

\section{THEORETICAL FORMULATION OF NONLINEAR BEAM OPTICS BY THE FILAMENT MODEL}

\section{A. Fundamental equation}

Beam-flattening systems with nonlinear optics have been studied via many approaches. Here, we describe a system with nonlinear beam optics and multipole magnets, as shown in Fig. 1. Linear elements such as quadrupole magnets are placed in the path of the nonlinear magnet and target, for example, at $s_{1}$ and $s_{t}$ in Fig. 1. If the nonlinear magnet for beam-profile transformation can generate an ideal nonlinear field that can be expanded into a power series of horizontal and vertical coordinates, $x$ and $y$, we have the following coupled nonlinear transverse equations of motion [42]:

$$
\begin{aligned}
& x^{\prime \prime}+K_{4}(s) x+\sum_{n=3}^{\infty} \frac{K_{2 n}}{(n-1) !} \Re\left[(x+i y)^{n-1}\right]+\cdots=0, \\
& y^{\prime \prime}-K_{4}(s) x+\sum_{n=3}^{\infty} \frac{K_{2 n}}{(n-1) !} \Re\left[i(x+i y)^{n-1}\right]+\cdots=0,
\end{aligned}
$$

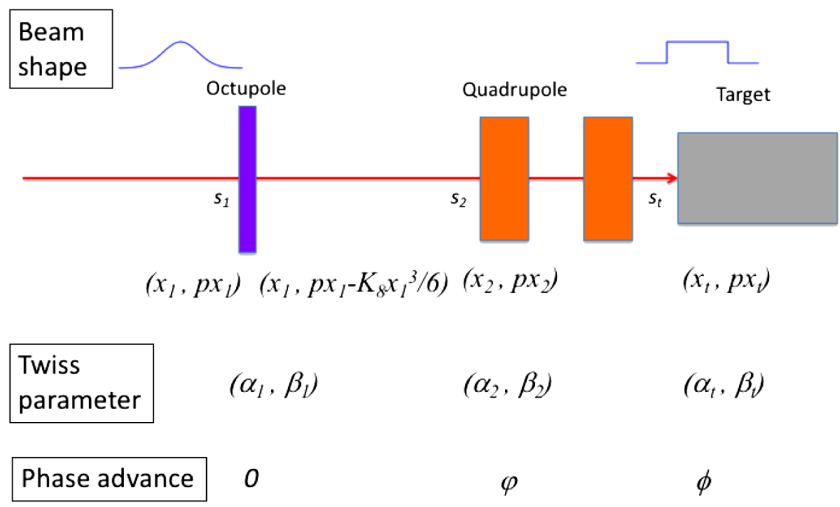

FIG. 1. Definition of beam transport parameters used in this study. 
where $K_{4}$ is the quadrupole strength and $K_{2 n}$ is the strength of the 2n-pole nonlinear component of the multipole magnet. The prime indicates differentiation with respect to path lengths. It is difficult to solve such a coupled equation of motion analytically. We thus expand the higher-order terms in Eq. (1) and obtain the following equations:

$$
\begin{array}{r}
x^{\prime \prime}+K_{4}(s) x+\frac{K_{6}}{2} x^{2}\left[1-\left(\frac{y}{x}\right)^{2}\right]+\frac{K_{8}}{3 !} x^{3}\left[1-3\left(\frac{y}{x}\right)^{2}\right]+\cdots=0, \\
y^{\prime \prime}-K_{4}(s) y+K_{6} x y+\frac{K_{8}}{3 !} y^{3}\left[1-3\left(\frac{x}{y}\right)^{2}\right]+\cdots=0 .
\end{array}
$$

To realize such a situation, we actually set the nonlinear magnet at the position where the beam size in one direction is sufficiently larger than that in the other, as demonstrated in Sec. IV. Therefore, one can focus only on one component of motion in the following. We focus on horizontal motion hereafter for the sake of simplicity. Using an octupole magnet, the beam at the target can be described as follows:

$$
\left(\begin{array}{c}
x_{t} \\
p x_{t}
\end{array}\right)=\left(\begin{array}{cc}
\sqrt{\frac{\beta_{t}}{\beta_{1}}}\left(\cos \phi+\alpha_{1} \sin \phi\right) & \sqrt{\beta_{t} \beta_{1}} \sin \phi \\
\frac{\left(\alpha_{1}-\alpha_{t}\right) \cos \phi-\left(1+\alpha_{t} \alpha_{1}\right) \sin \phi}{\sqrt{\beta_{t} \beta_{1}}} & \sqrt{\frac{\beta_{1}}{\beta_{t}}}\left(\cos \phi-\alpha_{t} \sin \phi\right)
\end{array}\right)\left(\begin{array}{c}
x_{1} \\
p x_{1}-K_{8} x_{1}^{3}\left[1-3\left(\frac{y_{1}}{x_{1}}\right)^{2}\right] / 6
\end{array}\right),
$$

where $x$ and $p x$ stand for the beam position and divergence at the target, respectively, with Twiss parameters $(\alpha$ and $\beta)$, and the phase advance $(\phi)$ shown in Fig. 1. $K_{8}$ is the integrated strength of the octupole magnet, where the magnet is assumed to be a thin lens for simplicity. Note that, for instance, in the horizontal expression of Eq. (3), vertical motion is approximately decoupled from horizontal motion for $|y / x| \ll 1$, particularly, in the region where the vertical amplitude is negligible compared to the horizontal one.

The beam position $x_{t}$ at the target can be expressed as

$$
x_{t}=\sqrt{\frac{\beta_{t}}{\beta_{1}}}\left(\cos \phi+\alpha_{1} \sin \phi\right) x_{1}+\sqrt{\beta_{t} \beta_{1}} \sin \phi p x_{1}-\sqrt{\beta_{t} \beta_{1}} \sin \phi K_{8} x_{1}^{3}\left[1-3\left(\frac{y_{1}}{x_{1}}\right)^{2}\right] / 6,
$$

for the $\left|y_{1} / x_{1}\right| \ll 1$ case, the above equation turns to be

$$
x_{t}=\sqrt{\frac{\beta_{t}}{\beta_{1}}}\left(\cos \phi+\alpha_{1} \sin \phi\right) x_{1}+\sqrt{\beta_{t} \beta_{1}} \sin \phi p x_{1}-\sqrt{\beta_{t} \beta_{1}} \sin \phi K_{8} x_{1}^{3} / 6 .
$$

\section{B. Filament-approximation model}

Normally, the beam is extended at the octupole magnet, and the divergence of the beam distribution is well described as $p x_{1}=-\alpha_{1} / \beta_{1} \cdot x_{1}$, where $\alpha_{1}$ and $\beta_{1}$ are the Twiss parameters of the octupole magnet. By this simplification, which is called the filament-approximation model in the present study, although the beam divergence is ignored, the beam distribution can be simplified using a filament with Gaussian amplitude at the octupole. As shown in the Appendix A, using the filament model, the target distribution can be described by the simplified thirdorder equation given below:

$$
x_{t}=\lambda_{1} x_{1}+\lambda_{3} x_{1}^{3}
$$

where $\lambda_{1}$ and $\lambda_{3}$ are

$\lambda_{1}=\sqrt{\beta_{t} / \beta_{1}} \cos \phi, \quad \lambda_{3}=-K_{8} \sqrt{\beta_{t} \beta_{1}} \sin \phi / 6$.

Since Eq. (6) is of the third order, it may have three distinct real roots if the discriminant formula is positive. If the discriminant formula is negative, Eq. (6) has a single real root. Moreover, Eq. (6) has multiple roots at $x_{t s}$, which can be obtained from the discriminant formula in Eq. (6),

$$
x_{t s}= \pm 2 / 3 \lambda_{1} \sqrt{-\lambda_{1} / 3 \lambda_{3}} \text {. }
$$

Given the differential of Eq. (6) with $x_{1}$ as zero (i.e., $d x_{t} / d x_{1}=0$ ), the beam position at the octupole magnet $\left(x_{1 s}\right)$ that yields multiple root points can be solved as 


$$
x_{1 s}= \pm \sqrt{-\lambda_{1} / 3 \lambda_{3}},
$$

if $\lambda_{1} / 3 \lambda_{3}$ is negative, singular points exist.

From the beam distribution at the octupole magnet, defined as $f\left(x_{1}\right)$, the beam distribution at the target, defined as $g\left(x_{t}\right)$, can be obtained by the conservation of integration particles of Eq. (6) as follows:

$$
g\left(x_{t}\right)=f\left(x_{1}\right) d x_{1} / d x_{t}=f\left(x_{1}\right) /\left|\lambda_{1}+3 \lambda_{3} x_{1}^{2}\right| .
$$

When $\left|x_{t}\right|<\left|x_{t s}\right|, x_{1}$ for each $x_{t}$ has double real roots; then Eq. (10) is described by the integration of each root. It should be noted that Eq. (10) yields infinite amplitude for singular points because the denominators are zero, as will be discussed in Appendix B.

\section{Octupole magnetic field}

As shown in Appendix $C$, a small value of the integrated octupole magnetic field of $K_{8}$ should be chosen to suppress beam loss. Substituting Eq. (7) into Eq. (8), we can find $K_{8}$ to obtain a flat shape as follows:

$$
K_{8}=8 \beta_{t} \cos ^{3} \phi / 9 x_{t s}^{2} \beta_{1}^{2} \sin \phi .
$$

By choosing a certain $\mathrm{x}_{t s}$ to fit the target size, $K_{8}$ is determined from Eq. (11). Here, two different approaches are considered to obtain an octupole magnetic field.

Méot and Aniel [29,30] studied nonlinear optics to obtain a uniform beam shape using octupole and dodecapole magnets. According to Ref. [29], for the singleoctupole-magnet case, the $x_{t s}$ required to obtain a flat distribution is empirically assumed to be $4 / 3 \sigma$, where $\sigma$ is beamwidth at the target for linear optics of $\sqrt{\epsilon_{\sigma} \beta_{t}}$ and $\epsilon_{\sigma}$ is one sigma of beam emittance. Therefore, the required magnetic field can be obtained as follows:

$$
K_{8}=\cos ^{3} \phi / 2 \epsilon_{\sigma} \beta_{1}^{2} \sin \phi=\cos ^{2} \phi / 2 \epsilon_{\sigma} \beta_{1}^{2} \tan \phi .
$$

Yuri et al. [23] studied nonlinear optics to obtain a flat distribution using infinite multipole magnets and the filament model, which is different from the approach adopted by Méot and Aniel. Using the Gaussian distribution in the initial condition of the transverse phase space, multipole magnetic fields up to infinity are determined to have completely flat shape at the target (see Appendix A). In Ref. [23], the half-width-beam flat region $(r)$ at the target was expressed as follows:

$$
r=\sqrt{\pi / 2} \sqrt{\epsilon_{\sigma} \beta_{t}}|\cos \phi| .
$$

If $r$ is chosen to be the singularity $\left(x_{t s}\right)$ in Eq. (11), $K_{8}$ is obtained as follows:

$$
K_{8}=16 / 9 \pi \epsilon_{\sigma} \beta_{1}^{2} \tan \phi \simeq 0.566 / \epsilon_{\sigma} \beta_{1}^{2} \tan \phi,
$$

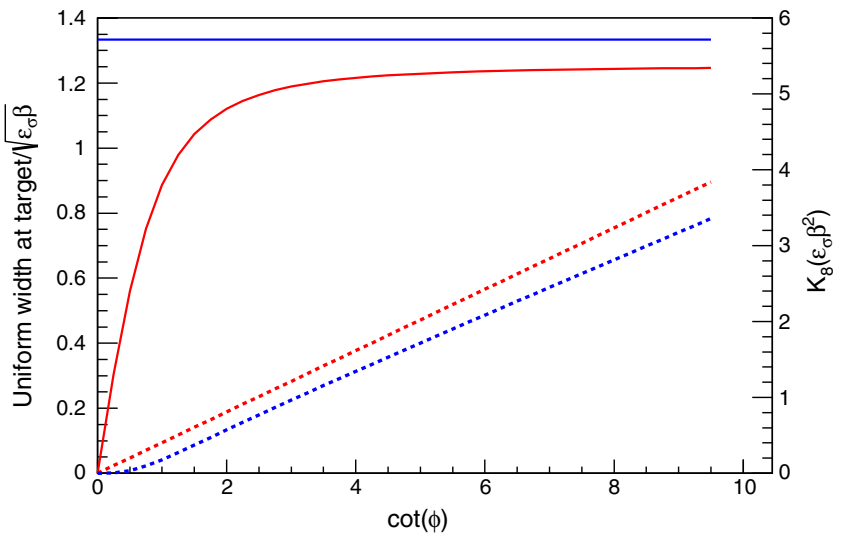

FIG. 2. Dependence of phase advance as a function of $\cot \phi$ for uniform beamwidth at target shown on the left-hand axis as solid lines described from Ref. [29] and Eq. (13) derived from Ref. [23], respectively, denoted by red and blue lines. The required octupole strength $\left(K_{8}\right)$ is shown on the right-hand axis as dotted lines, as obtained by calculation using Eqs. (12) and (14), respectively. On the vertical axis in the figure is shown the beamwidth $\sigma_{t}$ and the octupole strength $K_{8}$ multiplied by $1 / \sqrt{\epsilon_{\sigma} \beta_{t}}$ and $\epsilon_{\sigma} \beta_{1}^{2}$, respectively.

which is similar to Eq. (12) as obtained by the approach of Méot, except for the term $\cos ^{2} \phi$. It should be noted that the magnet's polarity is also given by Eqs. (12) and (14). Here, for the sake of simplicity, a positive case is discussed.

The required octupole strength of $K_{8}$ obtained with Eqs. (12) and (14) for various $\cot \phi$ is shown in Fig. 2. Although a slight discrepancy exists, the $K_{8}$ results obtained by Eqs. (12) and (14) are similar when $\cot \phi$ is larger than 4. In Fig. 2, a uniform beamwidth is shown for various phase-advance values. Because Méot's procedure fixes the beamwidth to $4 / 3 \sigma$ regardless of the phase advance $\phi$, the dependence of $\phi$ on uniform beamwidth does not appear, as shown in Fig. 2. By contrast, the beamwidth obtained using Yuri's procedure varies as a function of $\cos \phi$, as given by Eq. (13), so beamwidth increases drastically in the region with phase advance $\cot \phi<1$ (i.e., $\phi>\pi / 4$ ). In the region corresponding to $\cot \phi>1$, the beamwidth is saturated because of the increase in $\cot \phi$ and is closer to the result for larger $\cot \phi$ with Méot's assumption, according to which the beamwidth should be $4 / 3 \sigma$ to obtain a flat distribution at the target. It can be concluded that the results of Méot and Yuri are similar for the given region of phase advance in terms of $\cot \phi$ being larger than 4 .

\section{GENERAL MODEL FOR NONLINEAR BEAM OPTICS WITH BEAM TRACKING}

\section{A. Generalized beam transformation for the nonlinear optics with an octupole magnet}

The above discussion is made for the filament model. Since the filament model ignores the angular distribution at 
an octupole magnet, there is a limit to obtain precise transverse distribution downstream of the octupole. Therefore, it is difficult to considerably optimize the beam manipulation for mitigation of the target damage. Previous studies of nonlinear focus by an octupole have not discussed the general scheme by using the specified emittance and Twiss parameters. To comprehensively understand the nature of nonlinear focus, a precise transverse distribution on the target and the beam loss was obtained by tracking using the generalized scheme. For the general discussion that does not depend on the Twiss parameter and the beam emittance, the tracking was made using the normalized Gaussian function in the transverse phase space. The transverse distribution of $\left(x_{t}, p x_{t}\right)$ without the filament-approximation model at an arbitrary position downstream octupole magnet with phase advance $\phi$ is also given by Eq. (4).

With the position related to beam emittance of $\epsilon$ and Twiss parameter $\gamma_{1}$ at the octupole magnet, Eq. (5) turns to be for the $\left|y_{1} / x_{1}\right| \ll 1$ case,

$$
\begin{aligned}
x_{t}= & \sqrt{\epsilon \beta_{t}}\left(\cos \phi+\alpha_{1} \sin \phi\right)+\sqrt{\epsilon \gamma_{1} \beta_{t} \beta_{1}} \sin \phi \\
& -\frac{\sqrt{\epsilon \beta_{t}} \sin \phi}{6} K_{8} \epsilon \beta_{1}^{2} .
\end{aligned}
$$

For the case of a Gaussian in the phase distribution, the beam distribution is populated by the first and second terms shown in Eq. (15), which can be expressed by the Gaussian function with a width of one $\sigma_{t}$ given by $\left(\beta_{t} \epsilon_{\sigma}\right)^{0.5}$ for the achromatic beam line. Only the third term shown in Eq. (15) influences on the transverse nonlinear part given by an octupole magnet. Here, in order to express the general form of the beam distribution to be applied for arbitrary emittance and Twiss parameters, we introduce the following normalized octupole-magnet strength,

$$
K_{8}^{*} \equiv K_{8} \epsilon \beta_{1}^{2},
$$

where $K_{8}^{*}$ is the octupole-magnetic field normalized by the beam emittance and the square of the $\beta$ function at the octupole magnet. Note, the only positive case for $K_{8}^{*}$ is discussed for simplicity here. From Eq. (15), the distribution is found to be generalized using $K_{8}^{*}$ and $\phi$ for the nonlinear optics with an octupole magnet. As discussed in the filament model, the distortion by nonlinear optics is well explained as a function of $\cot \phi$, which is expected in the general model. By introducing both $K_{8}^{*}$ and $\cot \phi$, the distribution of nonlinear optics with an octupole magnet can be generalized and expressed with simplification.

To obtain the transverse beam distribution not only at the target, but also at another place downstream of the octupole magnet, we tracked the beam for various $\cot \phi$ and $K_{8}^{*}$ following Eq. (15). In the beam tracking, the values of $\cot \phi$ and $K_{8}^{*}$ were chosen up to 10 and 6 , respectively. In the calculation, the source particles are assumed to have a

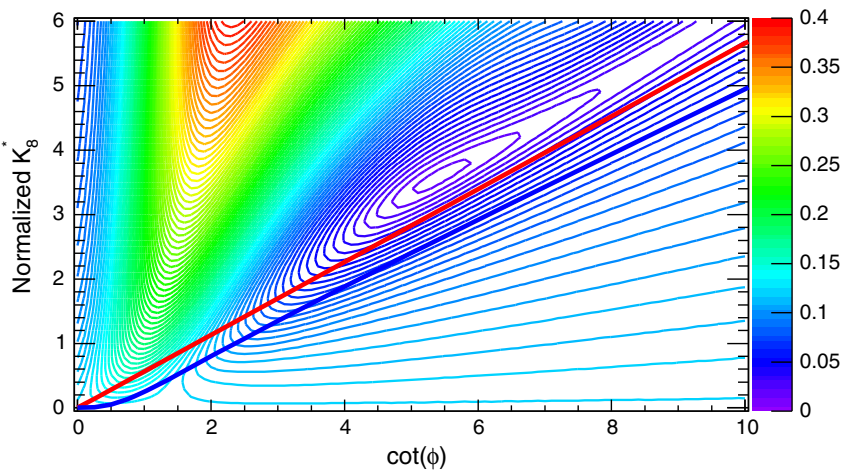

FIG. 3. Uniformity of the intensity in the beam position region within $\pm 1 \sigma_{t}$ shown as a function of normalized $K_{8}^{*}$ and $\cot \phi$. The solid blue and red lines are given by the filament model approximation by Eqs. (12) and (14), respectively.

Gaussian distribution in the phase space. To evaluate the small amount of the beam loss, a large number of source particles $2 \times 10^{11}$ was used in the calculation.

\section{B. Uniform-distribution case around at the center region}

Here, we discuss a flat distribution obtained the generalized form for nonlinear optics by using an octupole magnet without a filament model. From Eq. (13) given by the filament model, the uniform distribution is suggested to be within approximately $1 \sigma_{t}$. Therefore, the beamuniformity fluctuation in the beam-position region within about $1 \sigma_{t}$ was surveyed as a function of root-mean-square (rms). Figures 3 and 4 show the deviation of the beam intensity normalized to the peak intensity with the linearoptics case in the beam-position region within $\pm 1 \sigma_{t}$ and $\pm 1.1 \sigma_{t}$. Also, the parameters given from Eqs. (14) and (12) with the filament-model approximation are shown in Figs. 3 and 4. From those results, the best uniform distribution is achieved with the combination of the parameters $\left(K_{8}^{*}, \cot \phi\right)=(3.5,5.8)$ and $(7,4.2)$ in the

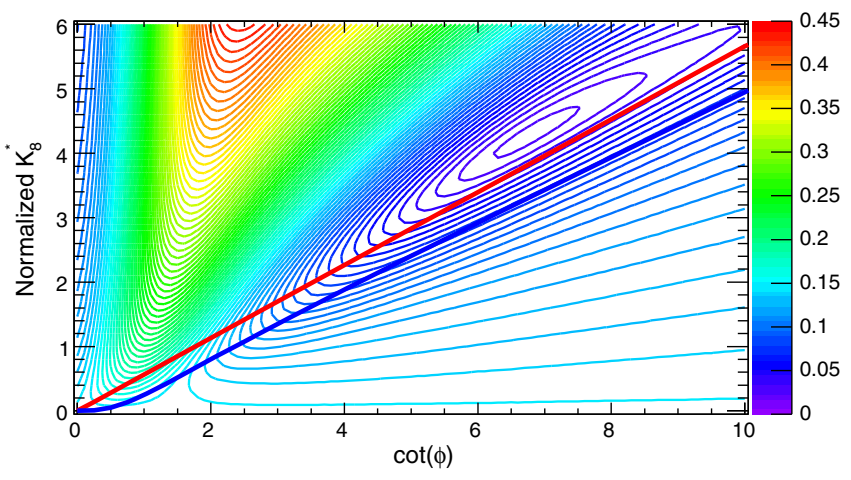

FIG. 4. Same as Fig. 3 but for the beam position region within $\pm 1.1 \sigma_{t}$. 


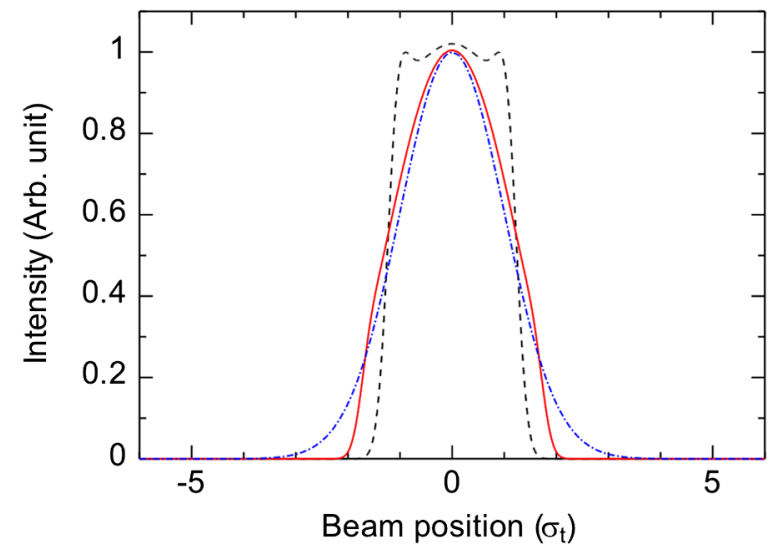

(a)

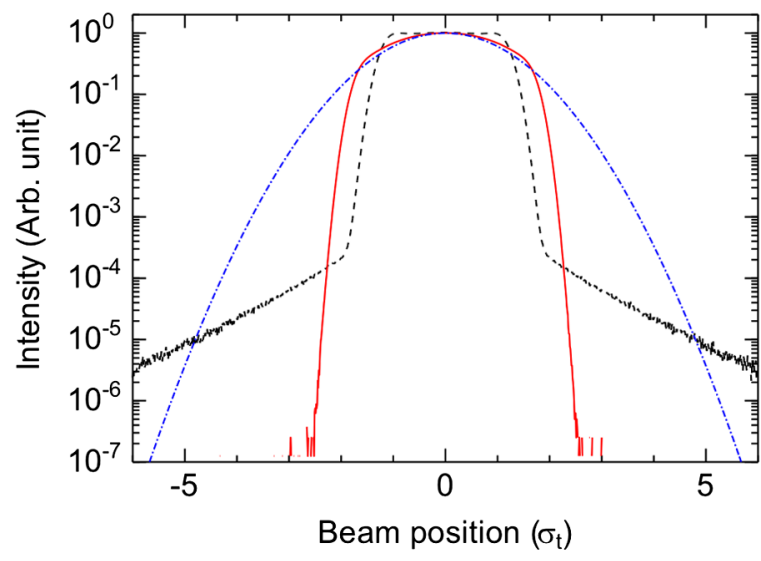

(b)

FIG. 5. Transverse beam distributions for the best flat case for $1 \sigma_{t}$ are shown as black dotted lines in parts (a) and (b) for linear and log scales, respectively. Also, the distribution with the minimum beam loss case for outside of $2.5 \sigma_{t}$ and Gaussian distribution with one $\sigma_{t}$ width are shown as solid red and dotted lines, respectively. The intensity is normalized by the peak intensity given by the linear optics.

region $\pm 1 \sigma_{t}$ and $\pm 1.1 \sigma_{t}$, respectively. It should be mentioned that the optimal parameter cannot be found through the filament model. By applying the generalized model, we can obtain the optimum parameters for nonlinear optics.

From the result shown in Figs. 3 and 4, it is found that the optimum parameters of $K_{8}^{*}$ and $\cot \phi$ increase following the increase of the area of interest according to $\sigma_{t}$. The filament model yields a large flat area from 1.2 to $1.3 \sigma_{t}$ shown in Fig. 2, which is larger than that employed in Fig. 4. In the present survey, we applied $\cot \phi$ up to 10 . If both larger $K_{8}^{*}$ and $\cot \phi$ were applied, the optimum values for $1.3 \sigma_{t}$ will be found; these are expected to have a larger $K_{8}^{*}$ than 6 and lie slightly above the red line shown in Fig. 4.

Figure 5 shows the beam profile for the best flat distribution for the region of $\pm 1 \sigma_{t}$. Although the distribution has a flat shape for the region between $-1 \sigma_{t}$ and $1 \sigma_{t}$, the intensity in the region above $5 \sigma_{t}$ turns out to be larger than for the Gaussian case with linear optics, as shown in Fig. 5(b). This phenomenon is caused by the spiral shaped in the phase space and rotation of the tail part opposed direction to the central one, as shown in Fig. 22. Eventually, the intensity at the tail corresponding to a large $\sigma_{t}$ becomes more substantial than that for the linear optics.

By placing the beam slit or collimator in front of the target with a slightly larger aperture than $\sim 1.1 \sigma_{t}$, one can obtain an almost completely flat distribution with the fluctuation of rms $\sim 3 \%$ eliminating the tail part. However, the present beam shaping with tail elimination can only be applied for the lowintensity accelerator case without the severe radio activation due to the beam loss. For a high-intensity hadron-accelerator case such as J-PARC, the elimination of the tail part is impossible due to the beam loss, which introduces radioactivity. Therefore, an additional method is required to obtain the low current density of the beam at the target discussed in Sec. III C.

\section{Manipulation to satisfy two antagonistic requirements for reduction of the beam-peak density and minimization of beam loss}

Using the nonlinear focusing, the shape of the beam can be manipulated to fit the aperture at the target as well. Due to the reduction of the intensity at the tail, it is possible to reduce the peak current density by expansion of the beamwidth with an increase of $\beta_{t}$. Here, we discussed a target with an aperture of $2.5 \sigma_{t}$ as a typical case. Figure 6 shows the integral intensity of the beam located outside of $\pm 2.5 \sigma_{t}$ for various $K_{8}^{*}$ and $\cot \phi$. Due to the nonlinear optics, the integral intensity can be found to be reduced about 7 magnitudes lower than linear optics with $K_{8}^{*}=1.5$ and $\cot \phi=8$, respectively. Note, the integral beam intensity for the same area with the linear optics is $1.2 \times 10^{-2}$. The integral intensity located outside of $2.5 \sigma_{t}$ becomes smaller for the case of $K_{8}^{*} \geq 0.5$ and $\cot \phi \geq 3$ due to nonlinear convergence. The solid black line shown in Fig. 6

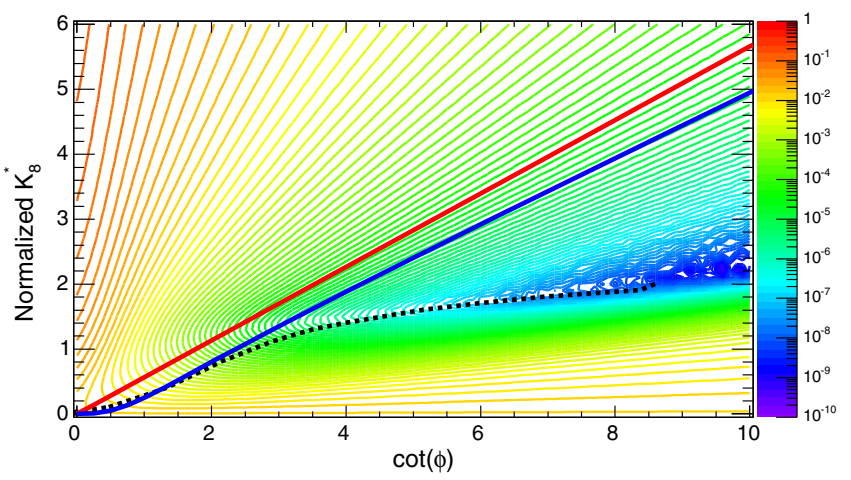

FIG. 6. Integral beam intensity to total at outside of $2.5 \sigma_{t}$ of linear optics and solid red and blue lines are the same as shown in Fig. 3. The black dotted line stands for the curve to be achieved minimum value for various $K_{8}^{*}$. 
indicates $\cot \phi$ is a guide curve to achieve the lowest intensity under minimization of $K_{8}^{*}$. In the figure, the results of the filament model are also shown in Fig. 2. For the same $K_{8}^{*}$ compared to the filament model, a slightly larger $\cot \phi$ was found to be optimal. Note, there was no significant difference in the optimal curve in the beam-width region between $2 \sigma_{t}$ and $3 \sigma_{t}$. Therefore, the solid black line in Fig. 6 can sustain beam convergence with minimized beam loss downstream of the octupole magnet. In Fig. 5, the transverse distribution is shown as a solid red line. The distribution is likely bell shaped and steeply converged at a position of $2.5 \sigma_{t}$. By choosing appropriate $2.5 \sigma_{t}$ just to fit the aperture placed in front of the target, one can reduce the peak current density.

To understand the beam-loss status along the beam line, the integral of the beam intensity was calculated for the large aperture. For instance, the aperture downstream of the octupole magnet was determined to have $6 \sigma$ for the linearoptics case, which is achieved at the MLF in J-PARC. In Fig. 7, the integral intensity is shown for the particle with the position located outside of $6 \sigma_{t}$. The maximum beam loss for various $K_{8}^{*}$ was found to be around $\cot \phi \sim-0.5$ as an increase of $K_{8}^{*}$. It is also found that the beam intensity outside of $2.5 \sigma_{t}$ increased in the region $\cot \phi<0$ along with $K_{8}^{*}$. In order to suppress beam loss due to divergence, it is crucial to select $K_{8}^{*}$ to be as low as possible.

As pragmatic use of high-intensity accelerators such as the spallation neutron source in J-PARC shown in the next session, which has an aperture of $6 \sigma$ of the linear optics, the beam loss due to nonlinear divergence is negligible for $K_{8}^{*} \sim 1$. From the black dotted line shown in Fig. 6, the condition of $K_{8}^{*} \sim 1$ and $\cot \phi \sim 3$ is considered to be almost ideal for decreasing the peak current density for the J-PARC case. Note, the allowable beam limit depends on the aperture from the octupole to the target. However, the black dotted line shown in Fig. 6 can be thought of as ideal for achieving the antagonistic requirements of reduction of the peak beam density and minimization of beam loss.

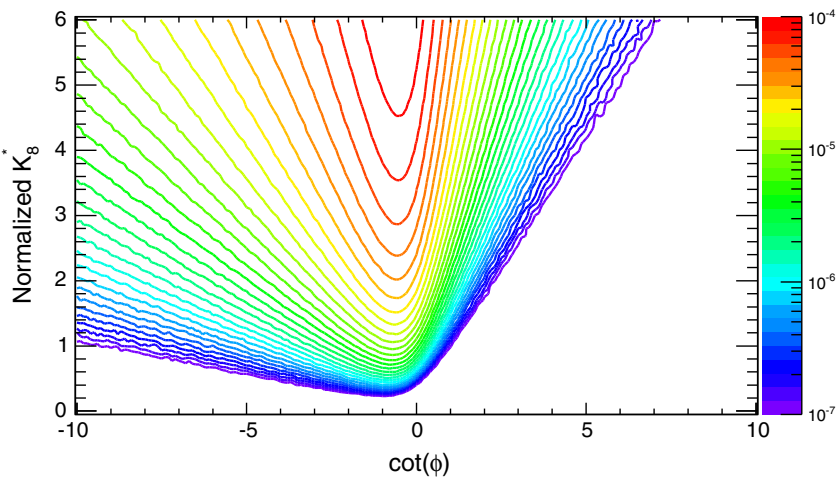

FIG. 7. Integral beam intensity to total at outside of $6 \sigma_{t}$ of linear optics.

\section{APPLICATION TO BEAM TRANSPORT AT THE SPALLATION NEUTRON SOURCE IN J-PARC}

\section{A. Beam transport from RCS to spallation neutron source in J-PARC}

At J-PARC, a 3-GeV proton beam with a beam power of $1 \mathrm{MW}$ and a repetition rate of $25 \mathrm{~Hz}$ is extracted from the RCS [43-45]. The $3-\mathrm{GeV}$ proton beam is delivered via a beam-transport channel called 3NBT in J-PARC, as shown in Fig. 8. To ensure it passes over the main ring, the beam is bent upward by a vertical angle of $7.5^{\circ}$. To fit the geometrical conditions in J-PARC, the beam is bent horizontally by $30^{\circ}$ using four bending magnets with each magnet bending it by an angle of $7.5^{\circ}$. In both bending sections, the optics is designed to achieve achromaticity.

The $3-\mathrm{GeV}$ proton beam extracted from the RCS is well described by a simple Gaussian $[43,46]$. With linear beam optics, the beam shape remains Gaussian at all positions along its path. Using nonlinear optics, the particles located at the beam periphery are deflected toward its center, which flattens the beam distribution. To obtain a flat distribution in both the horizontal and vertical directions, two octupole magnets are required. These octupole magnets can be placed anywhere upstream of the target, except where the phase advance between the magnets and the mercury target is an integer multiple of $\pi$. Because the targets have been irradiated by the proton beam for many years, the radiation dose around the targets is too high to place a magnet there. Therefore, the two octupole magnets (OCT1, OCT2) were placed upstream of the muon target, as shown in Fig. 9. OCT1 and OCT2 are used for beam focusing in the vertical and horizontal planes, respectively, by the nonlinear optics.

It should be noted that around the muon-production target, beam loss is expected to be about $8 \%$ due to the nuclear interaction. In designing the magnet, careful attention was paid to the insulator around the muon target because ordinal insulators such as polyimide for the coils

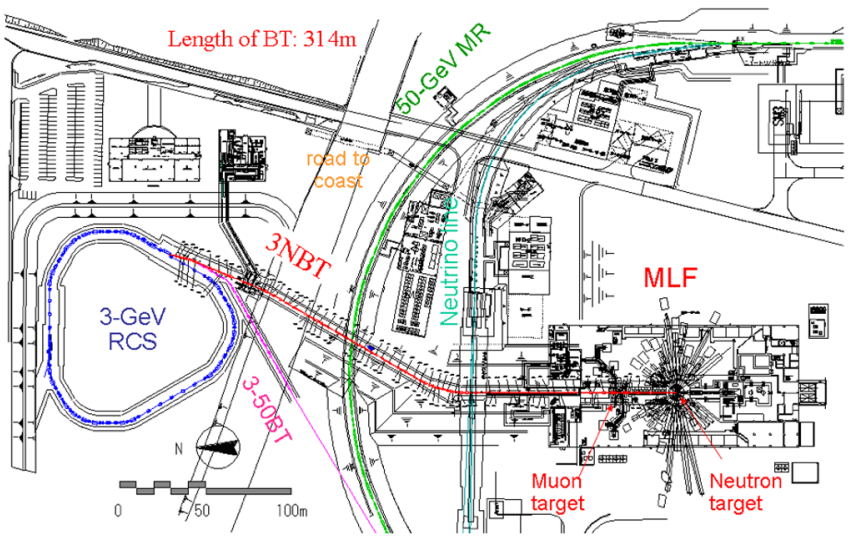

FIG. 8. Plan of rapid cycling synchrotron (RCS) at Materials and Life Science Experimental Facility (MLF) in J-PARC. 


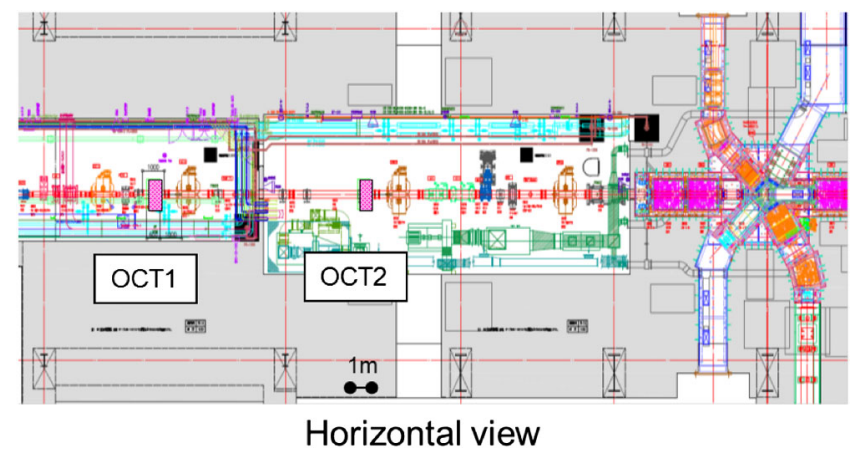

FIG. 9. Plan of octupole magnets and muon production target in J-PARC.

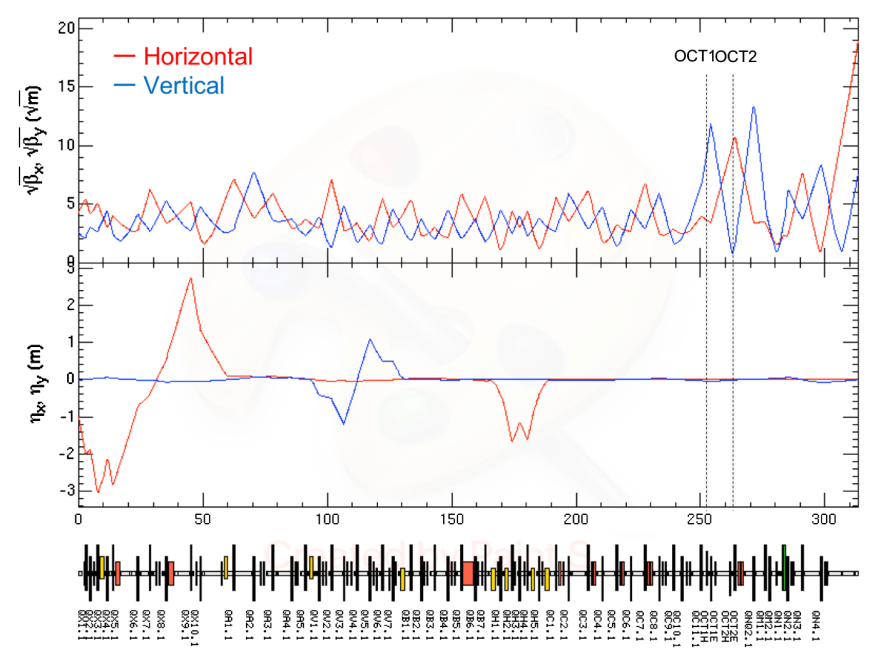

FIG. 10. Beam optics and beta function of nonlinear beam expander consisting of octupole magnets.
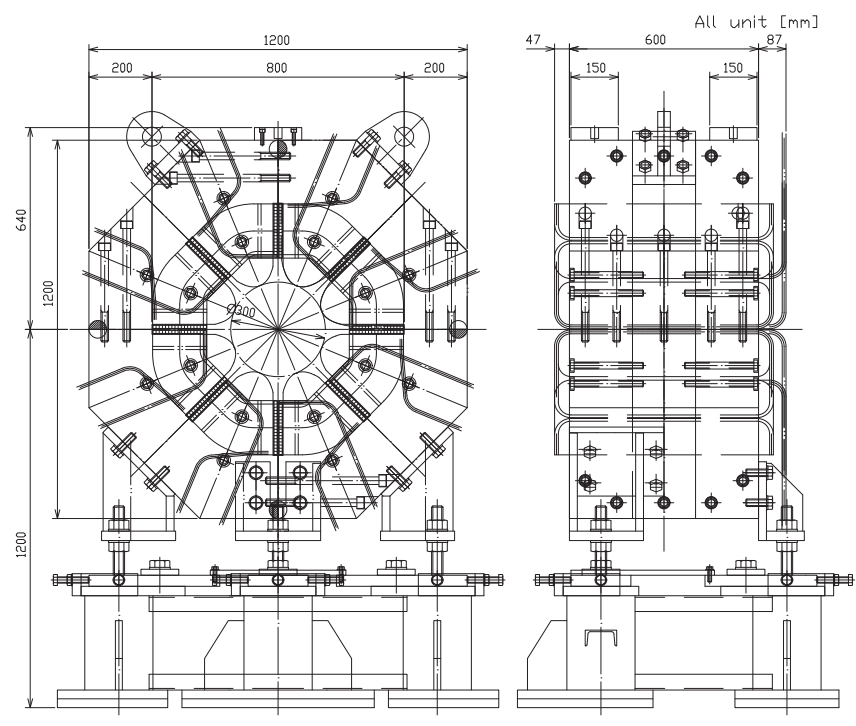

FIG. 11. Schematic drawing of fabricated octupole magnet.
TABLE I. Parameters of fabricated octupole magnet for nonlinear optics.

\begin{tabular}{lc}
\hline \hline Bore diameter $[\mathrm{mm}]$ & 300 \\
Pole length $[\mathrm{mm}]$ & 600 \\
Pole width $[\mathrm{mm}]$ & 262 \\
Field gradient & $\pm 928\left[\mathrm{~T} / \mathrm{m}^{3}\right]$ \\
& $\pm 43.6\left[/ \mathrm{m}^{2}\right]$ for $3-\mathrm{GeV}$ proton \\
Conductor cross section & Hollow conductor $\square 9 \mathrm{~mm}$ \\
& with cooling channel $\phi 6 \mathrm{~mm}$ \\
Insulator & Polyimide \\
Ampere turn [A $\cdot$ Turn $]$ & 14,000 \\
Winding number/coil & 20 \\
Maximum current $[\mathrm{A}]$ & \pm 700 \\
Temperature rise of water $\left[{ }^{\circ} \mathrm{C}\right]$ & 29.7 \\
Pole weight $[\mathrm{T}]$ & 4.85 \\
Coil weight $[\mathrm{T}]$ & 0.15 \\
Overall weight (except base) $[\mathrm{T}]$ & 5.0 \\
\hline \hline
\end{tabular}

will lose their insulation properties due to radiation. Therefore, a cable made of magnesia $(\mathrm{MgO})$ was used as a mineral insulator to withstand the high levels of radiation [47]. The magnets around the muon target can be replaced by remote handling to check for malfunction. The pin for alignment placed at the bottom of the magnet can determine the magnet's position. In the case of the magnets except other than those around the muon-production target, the allowable beam loss is $1 \mathrm{~W} / \mathrm{m}$ for hands-on maintenance. By introducing nonlinear optics, the induced beam loss does not increase significantly, as described below.

The beam optics for the entire beam-transport line is shown in Fig. 10, indicating the beta and dispersion functions from the RCS to the spallation neutron target. To achieve a flat beam distribution, the octupole field must be proportional to the inverse square of the beta functions described in Eq. (16). Because of the high momentum of the present beam, a large octupole field is difficult to achieve. To obtain a flat beam distribution with an octupole magnet having a realistic $K_{8}$, the beamwidth at the octupole

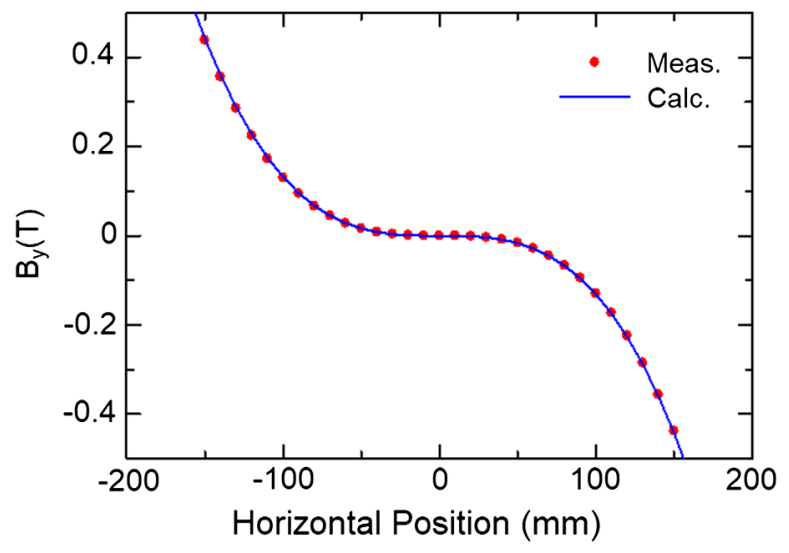

FIG. 12. Comparison of measured and calculated field gradient at octupole magnet for $800 \mathrm{~T} / \mathrm{m}^{3}$. 
TABLE II. Beam parameters of nonlinear optics used in the spallation neutron source in J-PARC.

\begin{tabular}{lcc}
\hline \hline Parameter & Horizontal & Vertical \\
\hline Beam emittance in one sigma $[\pi \mathrm{mm} \cdot \mathrm{mrad}]$ & 5.24 & 6.43 \\
Octupole strength $K_{8}\left[/ \mathrm{m}^{2}\right]$ & -43.4 & -43.4 \\
Normalized strength $K_{8}^{*}$ & -1.8 & -2.2 \\
$\beta$ at octupole $(\mathrm{OCT} 2)$ for horizontal focus $[\mathrm{m}]$ & 89.2 & 1.8 \\
$\alpha$ at octupole $(\mathrm{OCT} 2)$ for horizontal focus & -0.06 & -11.9 \\
$\beta$ at octupole (OCT1) for vertical focus [m] & 9.8 & 88.9 \\
$\alpha$ at octupole (OCT1) for horizontal focus & -12.0 & 1.36 \\
$\beta$ at neutron target $[\mathrm{m}]$ & 361.3 & 67.0 \\
Phase advance $\phi[\mathrm{deg}]$ & -17.4 & 14.4 \\
Phase advance as $\cot \phi$ & -3.2 & -3.9 \\
\hline \hline
\end{tabular}

magnet is increased by increasing $\beta$ to a large value. The aperture of the quadrupole magnets was fixed at $300 \mathrm{~mm}$. Therefore, we set the aperture of the octupole magnets to $300 \mathrm{~mm}$. For linear beam optics, beam admittance is designed to be $324 \pi \mathrm{mm} \cdot \mathrm{mrad}$, which is given by the beam collimator placed in the RCS. A recent study of the RCS shows that the transverse emittance can be reduced to as little as $250 \pi \mathrm{mm} \cdot \mathrm{mrad}$ [48]. Therefore, beam admittance at the octupole magnet must be $250 \pi \mathrm{mm} \cdot \mathrm{mrad}$, and accordingly, $\beta$ was set to $\sim 90 \mathrm{~m}$ at the octupole magnets.

\section{B. Octupole magnet}

Based on the optical design, the two pieces of the octupole magnet shown in Fig. 11 were fabricated for horizontal and vertical nonlinear focus on the target. To meet the requirements, we evaluated the saturation field of the octupole magnets with a three-dimensional fieldanalysis code (OPERA-3D). The parameters of the present octupole magnet are listed in Table I. The designed magnetic field gradient is $\sim 900 \mathrm{~T} / \mathrm{m}^{3}$, and the magnet has a bore diameter of $0.3 \mathrm{~m}$ and a length of $0.6 \mathrm{~m}$ in the direction of the pole. Using a Hall probe, the field gradient of the present octupole magnet was measured, as shown in Fig. 12. The magnetic field calculated using OPERA-3D is also shown in Fig. 12 for comparison with the measured value, and the two values agree well. The polarity of the octupole is determined as $\cot \phi$, which is given by the phase advance. To obtain a large degree of freedom for beam turning, the octupole magnet was powered using bipolar power supplies. During actual beam operation, beam centering at the octupole is important. To center the beam, a beam-position monitor (BPM) was installed in each magnet.

\section{Calculation of beam profile and loss by nonlinear optics}

To calculate the beam profile at the neutron source, we used the SAD code and specified initial beam conditions such as transverse emittance and Twiss parameters by fitting the beamwidth observed by the multiwire-profile monitors (MWPM). For multiple scattering at the muon target, the DECAY-TURTLE [49] code revised by the Paul Scherrer Institute (PSI) [50] was implemented in the SAD code [51]. The beam loss owing to multiple scattering at the muon-production target was shown to be $8 \%$, irrespective of the type of linear optics.

Beam loss due to the beam envelope at the tail was calculated with SAD based on the beam tracking result of the generalized model, when using the beam optics summarized in Table II. In Fig. 13, the beam envelope by nonlinear optics is shown for the relative intensity to the total. The beam envelope of linear optics having the intensity of $10^{-8}$ is also shown as cyan lines in Fig. 13. The horizontal beam was found to be enlarged at the quadrupole magnets QM1 and QN3, owing to defocusing by the nonlinear optics. However, the beam loss due to the nonlinear optics in the case of the present beam transport, in which the half-aperture size is $140 \mathrm{~mm}$, is on the order of

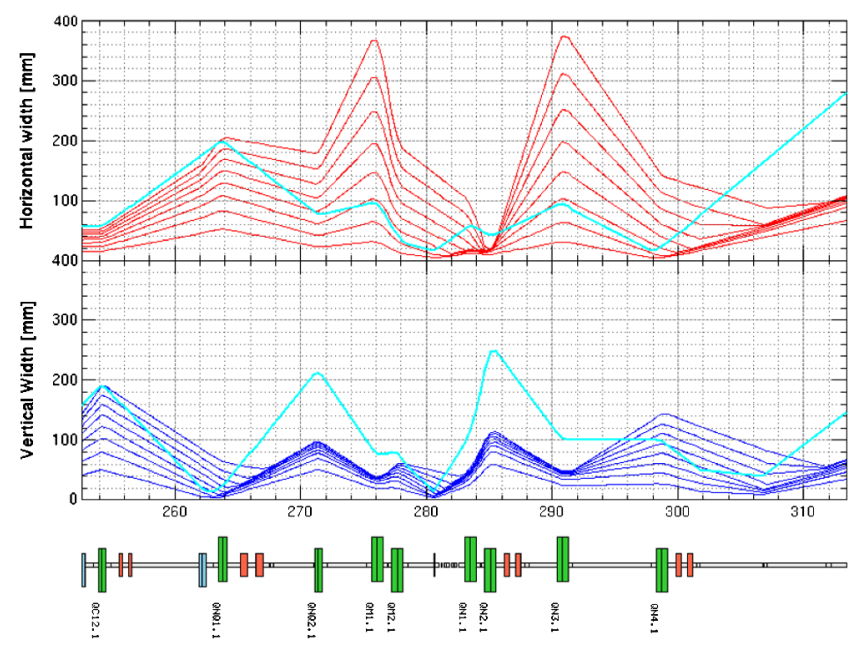

FIG. 13. Calculation of the beam envelope by nonlinear optics in horizontal (red line) and vertical planes (blue line) shown at the top and the bottom, respectively. Each line shows the envelope for each decade from $10^{-8}$ to $10^{-1}$ of the total intensity, compared with the envelope obtained using linear optics for $10^{-8}$ of the total intensity. 


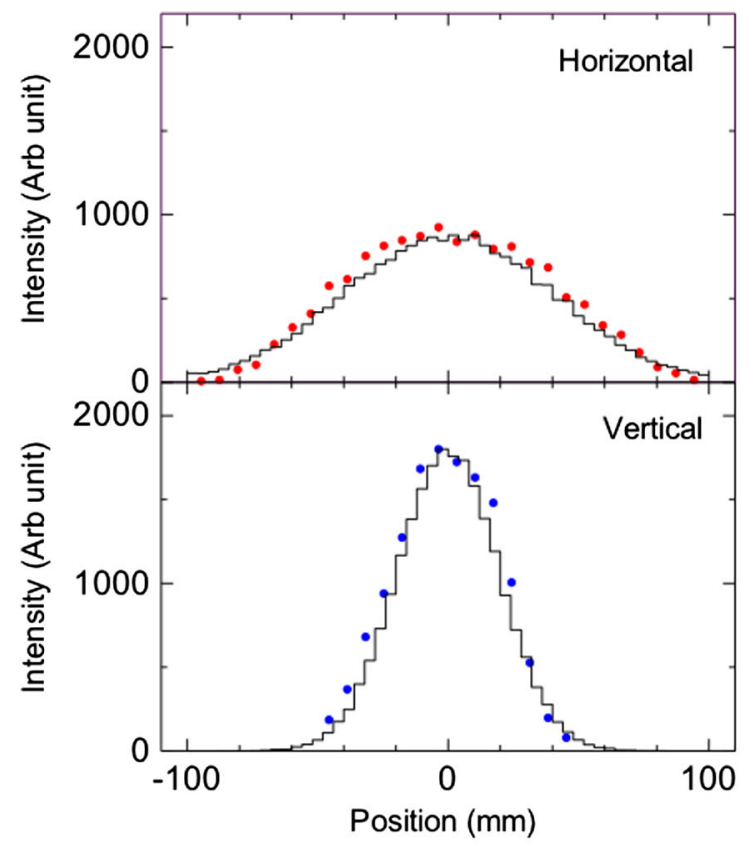

(a)

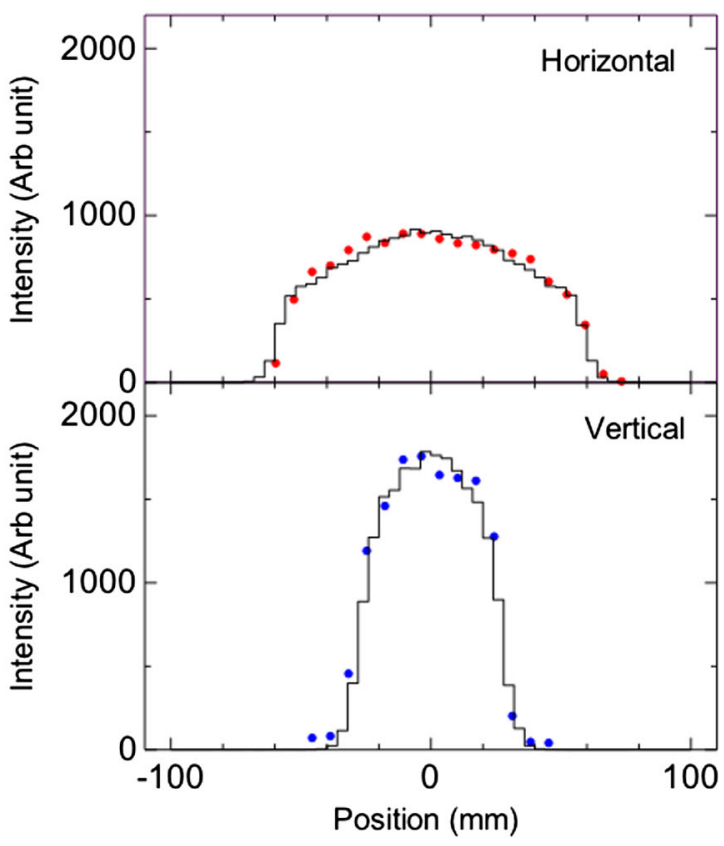

(b)

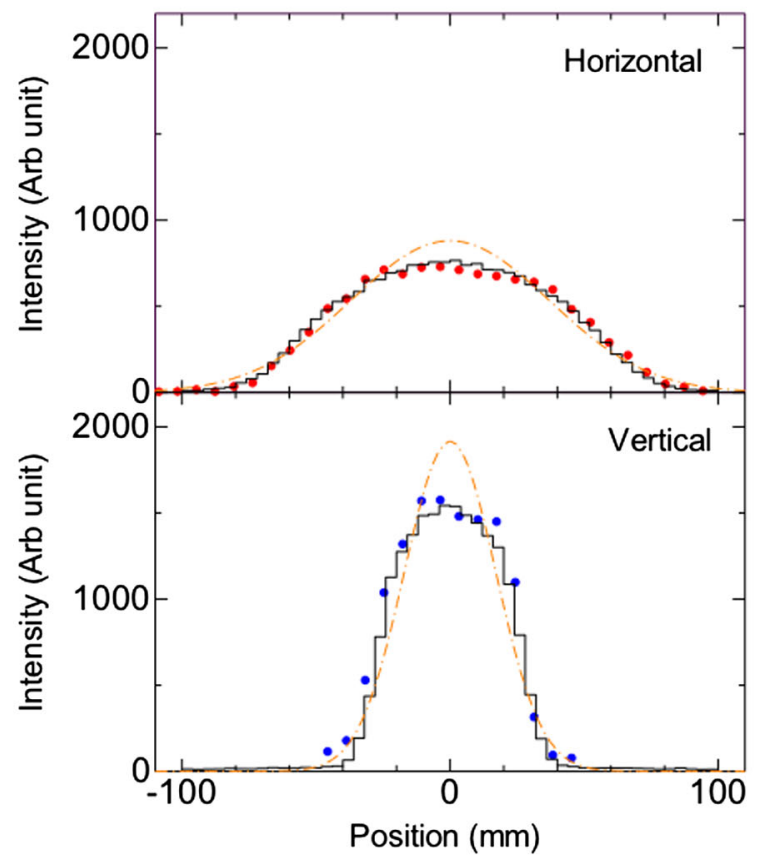

(c)

FIG. 14. Beam profile of 1-MW beam obtained with MWPM placed at PBW in the horizontal and vertical planes shown at the top and bottom, respectively, where (a) and (b) show the results for the cases of linear and nonlinear optics with current of 698 A supplied to the octupole magnet, and comparison with the calculation result obtained with tracking using SAD. Panel (c) shows the result for nonlinear optics placing with the muon production target compared with simulation scattering effect by DECAY-TURTLE [50] and compared with the linear optics result (dotted orange line) allowable for beam operation.

$10^{5}$ and is considerably lower than the beam loss due to scattering at the muon-production target. Hence, the beam loss due to nonlinear defocusing is acceptable. The radiation-dose measurement after beam operation indicates that the activation did not increase significantly with the introduction of nonlinear optics. The rate after 0.5 MW operation for one month was observed by a small radiation dosimeter placed about $30 \mathrm{~cm}$ from the magnet 
QN3, which was placed downstream of the muon production target. For the cases with and without nonlinear optics, the dose rate was about $30 \mathrm{mSv} / \mathrm{h}$, which implies a constant beam irrespective of the use of nonlinear optics. Although a slight increase in the dose rate was found from 10 to $30 \mu \mathrm{Sv} / h$ around the octupole magnet due to the use of nonlinear optics, this increase was insignificant, and the loss was equivalent at a level of $10 \mathrm{~mW}$, consistent with the result shown in Fig. 13 and allowing for high-intensity beam operation such as $1 \mathrm{MW}$.

Note that the beam loss shown in Fig. 13 was calculated ignoring the decoupling of horizontal and vertical at the octupole magnet. At the horizontal-focusing octupole

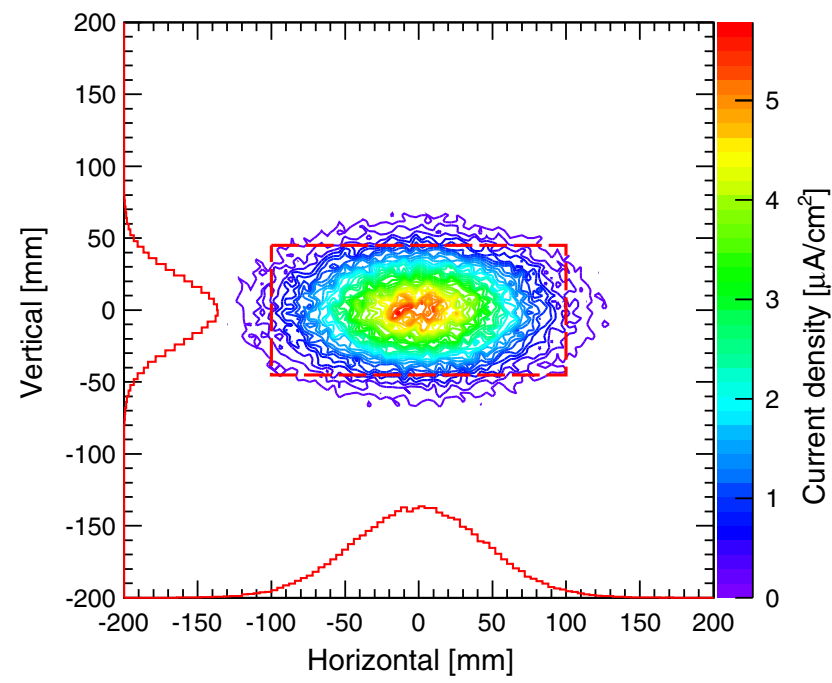

(a)

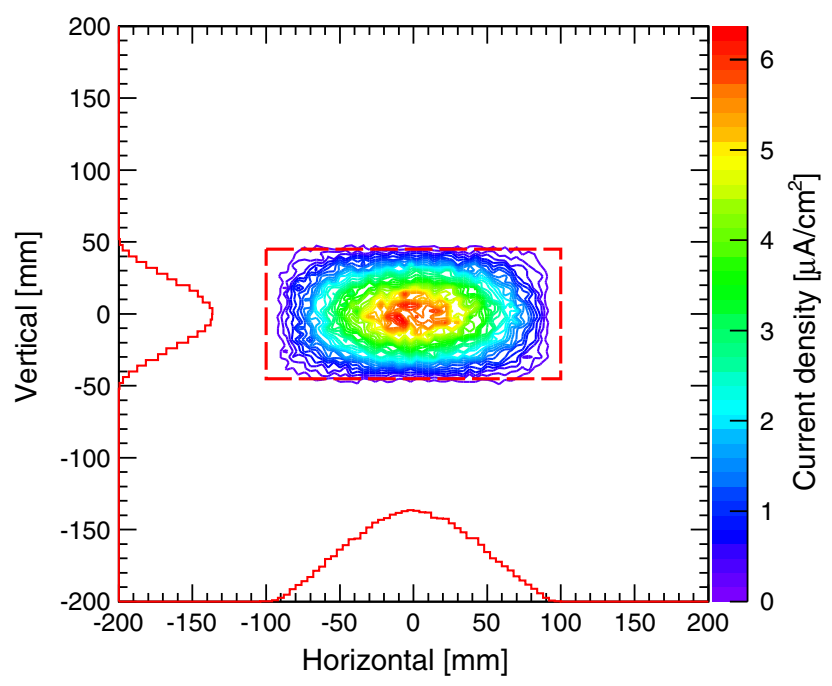

(c)
(OCT2), the $\beta_{y}$ of the vertical, shown in Table II, is relatively large, which is $\sim 10 \%$ of the horizontal one. As shown in Eq. (4), the influence of $\beta_{y}$ to the beamwidth at downstream can be treated as the strength of $K_{8}$ decreased with proportional of $3\left(y_{1} / x_{1}\right)^{2}$. For the case shown in Table II, the vertical width for $1 \sigma$ at the OCT2 decreases only $~ 5 \%$ of $K_{8}$ for horizontal width for $1 \sigma$. The decreasing factor of $K_{8}$ for the OCT2 becomes proportional to the inverse square of the beamwidth in the horizontal direction. Therefore, the beam loss with the coupling slightly decreases from one without the coupling. Since the beam loss following the octupole magnet is mainly caused by the beam having large $x_{1}$, the beam loss status shown in Fig. 13 can be thought to predict well.

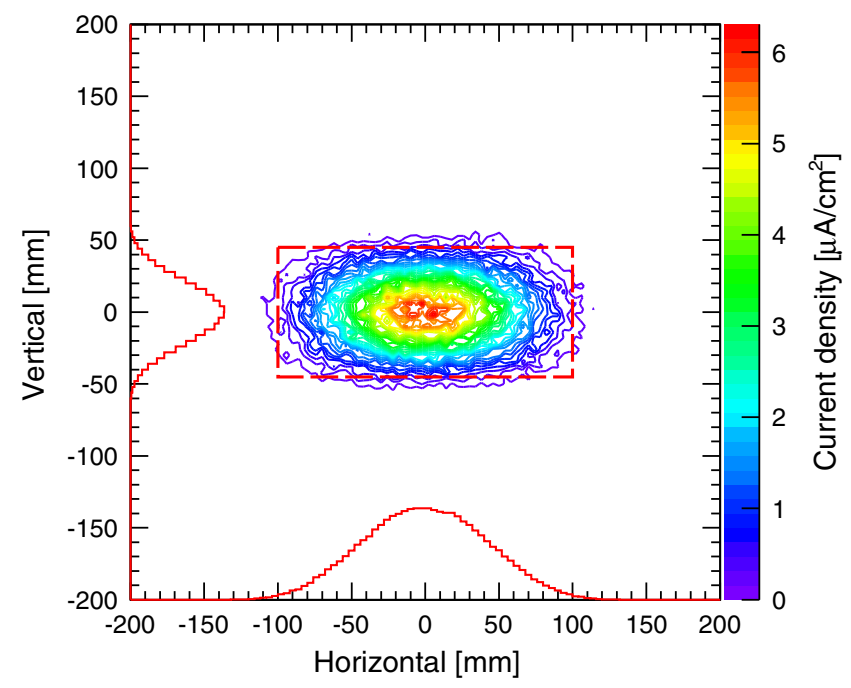

(b)

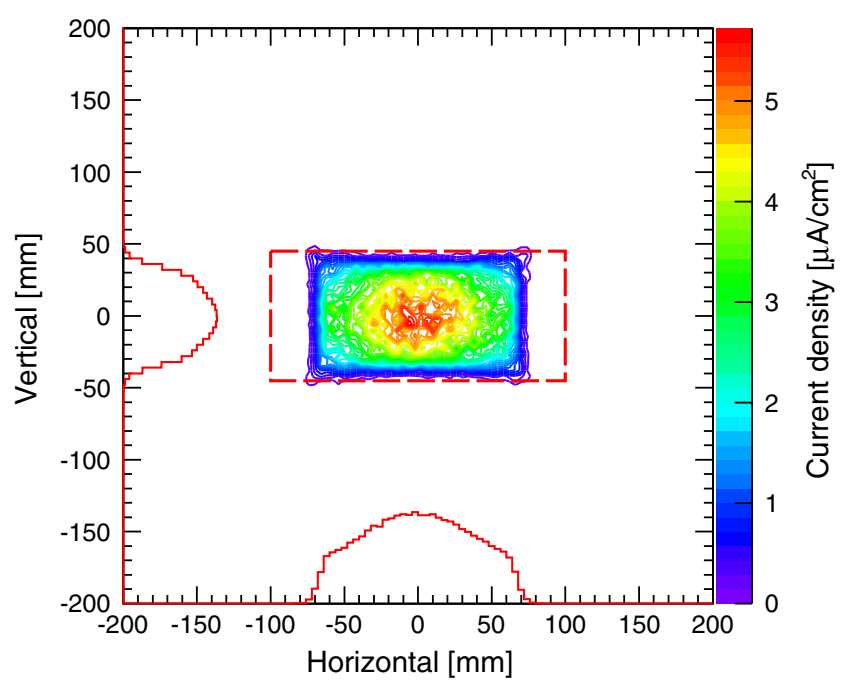

(d)

FIG. 15. Calculated beam profile at neutron production target for various currents supplied to octupole magnet without interaction with muon production target. Each panel shows both contour profile and projection profile on each plane. Panels (a)-(d) show the results for octupole currents of 0, 200, 400, and 698 A, respectively. 


\section{Beam profile at the spallation neutron source in J-PARC}

When using the beam optics summarized in Table II, the obtained beam profile with tracking is compared with the experimental results. In the calculation, we took into account the momentum spread of the beam $\delta p / p \sim$ $0.2 \%$ observed at the RCS [52]. Also, the coupling effect of the beam at the octupole magnets was considered without simplification of the matrix for the octupole. Since a negative sign was appended to the term $\cot \phi$, a negative value of $K_{8}$ is used. Figure 14 shows the profile results for the 1-MW beam observed using a MWPM placed in the proton-beam window (PBW) [46], which is 1.8-m upstream of the mercury target.

Figures 14(a) and 14(b) show the profile results for the beam profiles with and without nonlinear optics (i.e., with and without excitation by the octupole magnet), respectively. The calculation results obtained with and without nonlinear optics are also shown in Fig. 14. The calculation results show remarkably good agreement with the experimental results in both cases. Figure 14(c) also compares the profile with the muon target using nonlinear optics, and again, the calculation results show remarkably good agreement with experiment. It can be said that by using the present calculation scheme, the beam profile can be predicted reliably.

The profiles of the 1-MW beam obtained with MWPM placed at PBW in the horizontal and vertical planes are shown at the top and bottom, respectively, where (a) and (b) show the results for the cases of linear and nonlinear optics with a current of 698 A supplied to the octupole magnet, and a comparison with the calculation result obtained via tracking using SAD. Panel (c) shows the result for nonlinear optics placing with muon production target compared with simulation scattering effect by DECAYTURTLE [50] and compared with the linear optics result (dotted orange line) allowable for beam operation.

To understand beam-profile dependence on the strength of the octupole magnet, the calculated profiles at the target for various strengths are shown in Fig. 15. Since the twodimensional beam profile monitor is not placed in front of the spallation neutron target, only the calculation results are shown in Fig. 15. The beam aperture at the entrance of the target with a shield in front of the target is also shown as a red rectangle at the center of Fig. 15. For the linear-optics case shown in Fig. 15(a), the beam is larger than the aperture of the shield, and this is not useful for practical beam operation because of the excessive heat load it causes near the target. With increasing octupole strength, the beamwidth is found to shrink, owing to focusing by the nonlinear optics; and eventually, the tail of the beam is placed inside the aperture, which is acceptable from the viewpoint of practical beam operation. In Fig. 16, the beam profile at the neutron source obtained by placing the muonproduction target is shown. Although the beam shape is

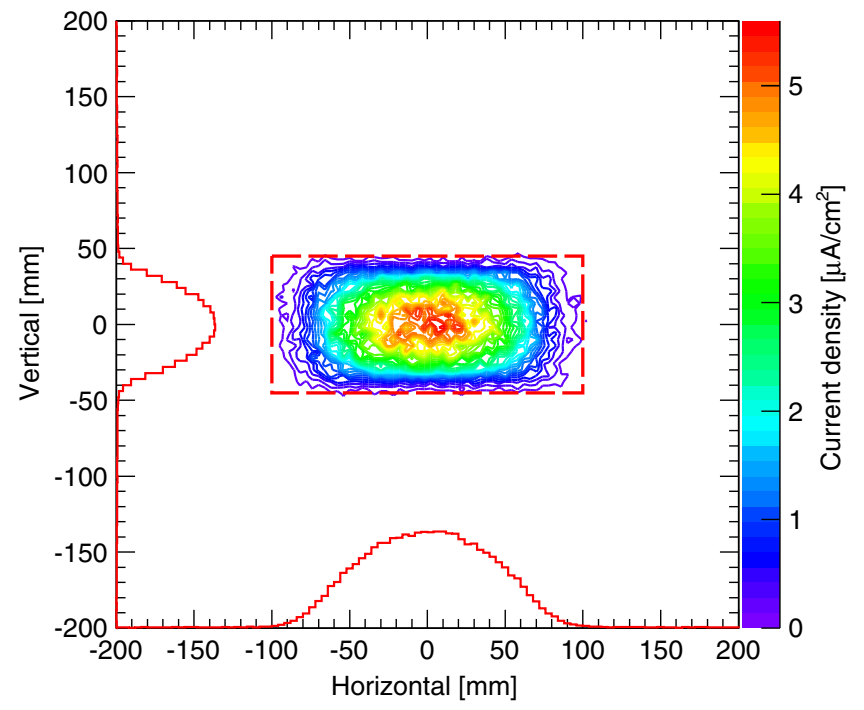

FIG. 16. Calculated beam profile at neutron production target with octupole magnet and muon production target.

Gaussian owing to scattering at the muon-production target, its bell shape, which fills the overall aperture, is retained. As a matter of fact, the peak current density is about $6 \mu \mathrm{A} / \mathrm{cm}^{2}$, which can reduce half of the density for the linear-optics case with an acceptable beam-heat deposition outside of the target.

To confirm the effect of nonlinear optics on the muonproduction target, we calculated the beam profile at the muon target for the various magnetic fields of the octupole. Figure 17 shows the calculated beam profile on the target for muon production. As the strength increases, the horizontal and vertical sizes increase slightly and decrease, respectively, owing to the nonlinear defocusing and focusing. It should be noted that this tendency is also shown in Fig. 12. Because the size difference caused by the introduction of nonlinear optics is negligible, the present optics is acceptable for muon production.

\section{E. Effect of misaligned magnets upon the beam profile}

In actual beam operation, a misaligned magnet causes beam divergence. A geometrical survey showed that the floor of the beam line facility settled unevenly after the Great East Japan earthquake happened on March 11, 2011, and the water table underlying the tunnel shifted [53,54]. Therefore, we cannot avoid beam divergence. In this section, we discuss the effect of misalignment on the beam profile based on the beam optics shown in Fig. 17. To simplify the calculation, the beam profile was calculated without scattering at the muon target.

To identify the alignment tolerance of the octupole magnet, the beam profile at the target was calculated via the beam-position offset at the octupole magnet. Without shifting the beam at the octupole magnet, its distribution is flat. For a horizontal offset of $2 \mathrm{~mm}$ in the beam position at 


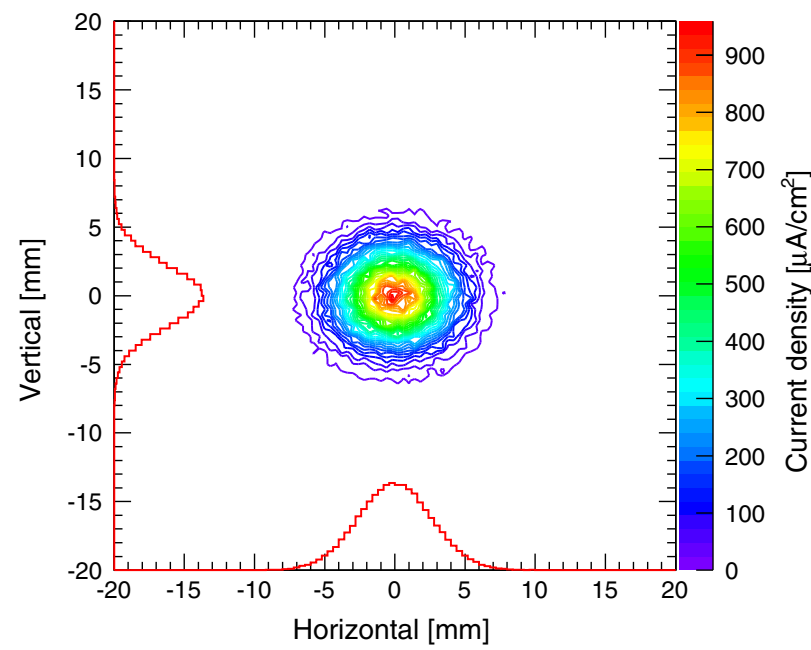

(a)

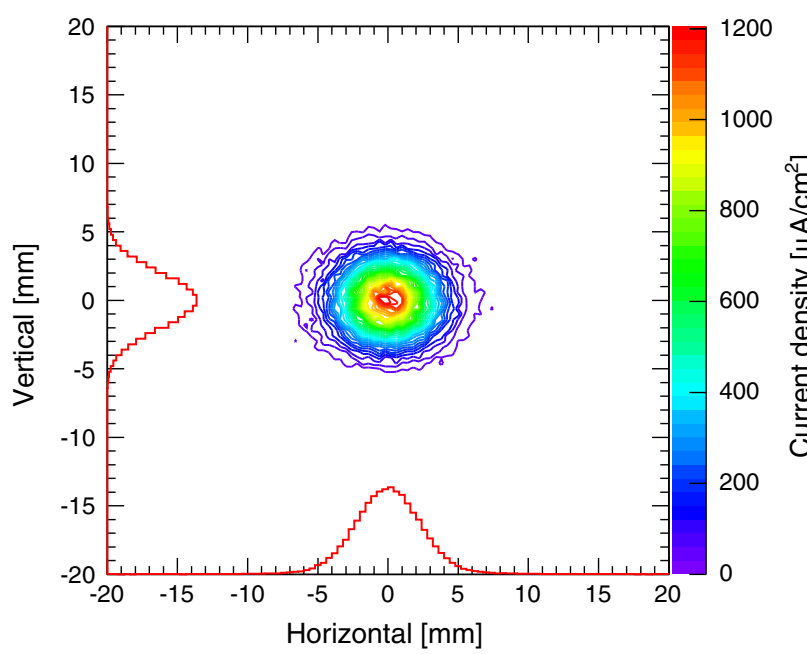

(c)

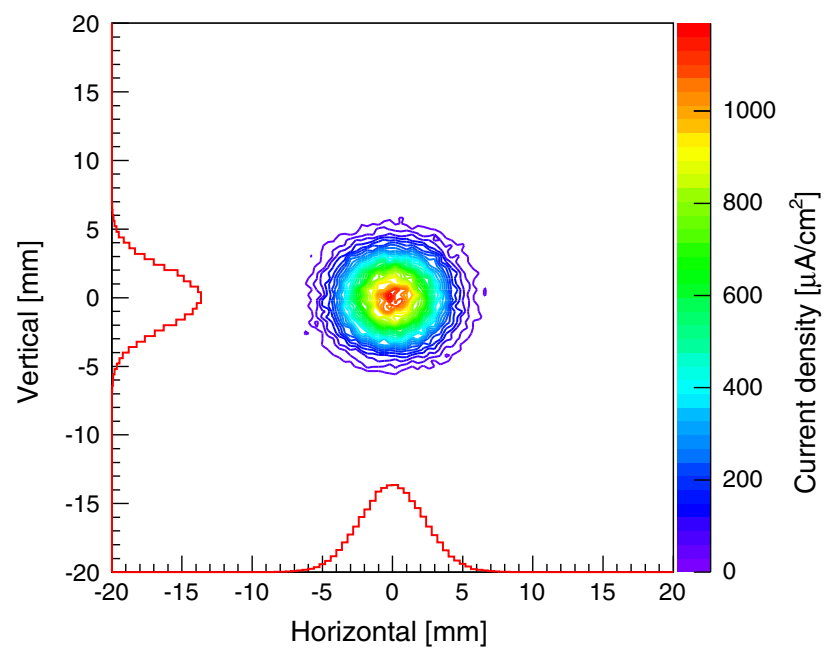

(b)

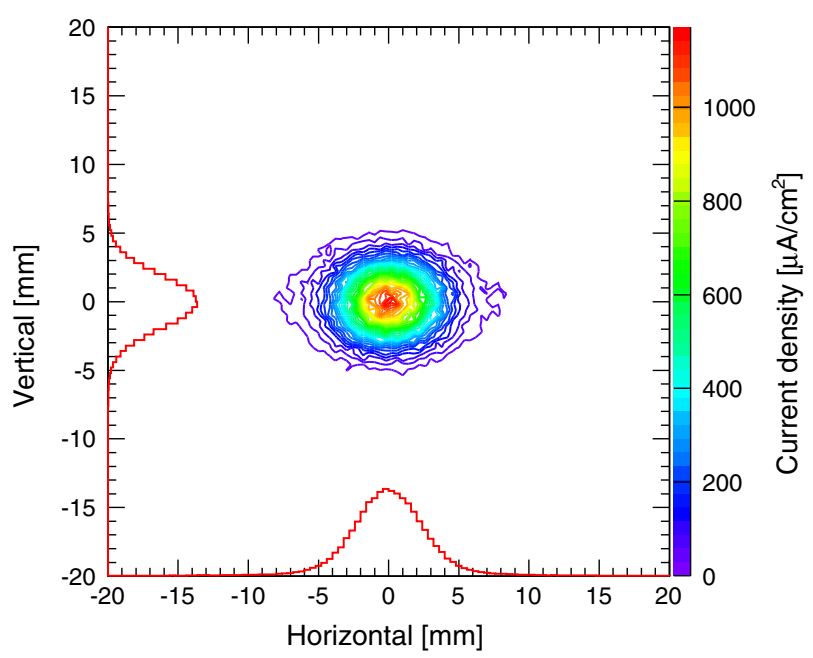

(d)

FIG. 17. Calculated beam profile at muon production target for various strengths of octupole magnet. The notations of (a)-(d) are as those in Fig. 15.

the octupole magnet, the beam shape has a peak at the beam edge, where intensity increases by approximately $8 \%$, as shown in Fig. 18. At the mercury target, the peak at the edge causes more significant damage than the peak at the center. The maximum increase in intensity at the edge is considered to be approximately $4 \%$. Therefore, the allowable beam shift at the octupole magnet is approximately $1 \mathrm{~mm}$. To adjust the beam position at the octupole magnet, BPMs will be installed. By installing BPMs and additional steering magnets, the beam position can be centered statically there. In each beam shot, a fluctuation in the horizontal position may occur because of the instability of the kicker magnet at RCS. However, the position instability for each shot is less than $1 \mathrm{~mm}$, so the edge peak does not cause problems.
To calculate the tilting tolerance of the octupole magnet, we considered the case of $0.5 \mathrm{mrad}$. We observed that the tilting error does not influence the beam shape. Because the precision of the angular position of the octupole magnet is less than $0.2 \mathrm{mrad}$, tilt misalignment is not a problem. Downstream of the octupole magnets, six quadrupole magnets were positioned around the muon production target. To obtain the required position accuracy of the downstream magnets, beam profiles were calculated for the quadrupole magnets with beam offset. We found that misalignment of the quadrupole magnets led to oscillation of the beam position because of betatron oscillation. Thus, the misalignment of the quadrupole magnets does not influence the beam shape, and the flat beam distribution at the target is preserved. Because the beam position at the 


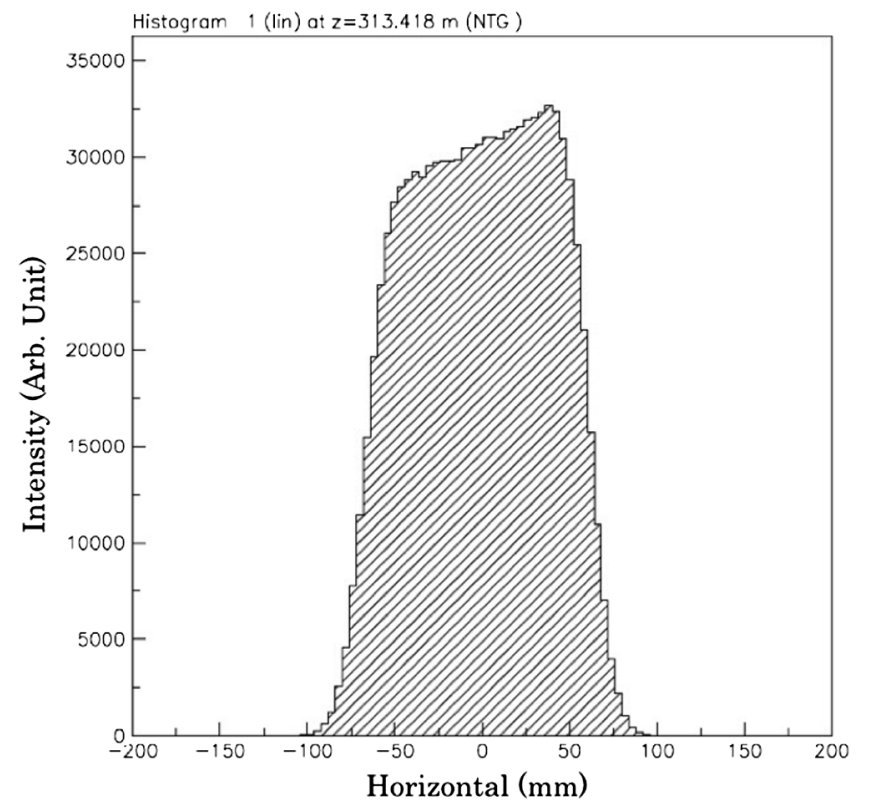

FIG. 18. Horizontal beam profile at neutron production target for $2 \mathrm{~mm}$ horizontal beam offset at the octupole magnet for horizontal focus (OCT2).

target can be easily adjusted using steering magnets, misalignment of the quadrupole magnets downstream of the octupole magnets does not affect beam flattening.

\section{F. Effect of beam scattering at the muon-production target}

To minimize the peak density and obtain a flat beam distribution at the neutron-production target, we studied the effect of beam scattering from this target. For the octupole magnets in use, the beam distribution in the horizontal phase space, prior to penetrating the carbon target, is shown

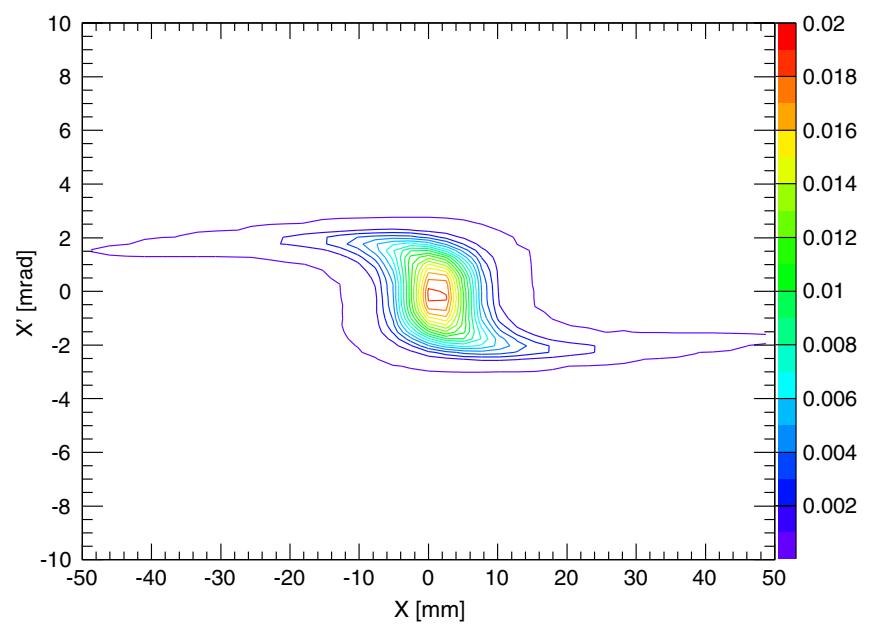

FIG. 19. Contour plot of transverse phase space distribution in the horizontal direction for beam injected into the muon production target.

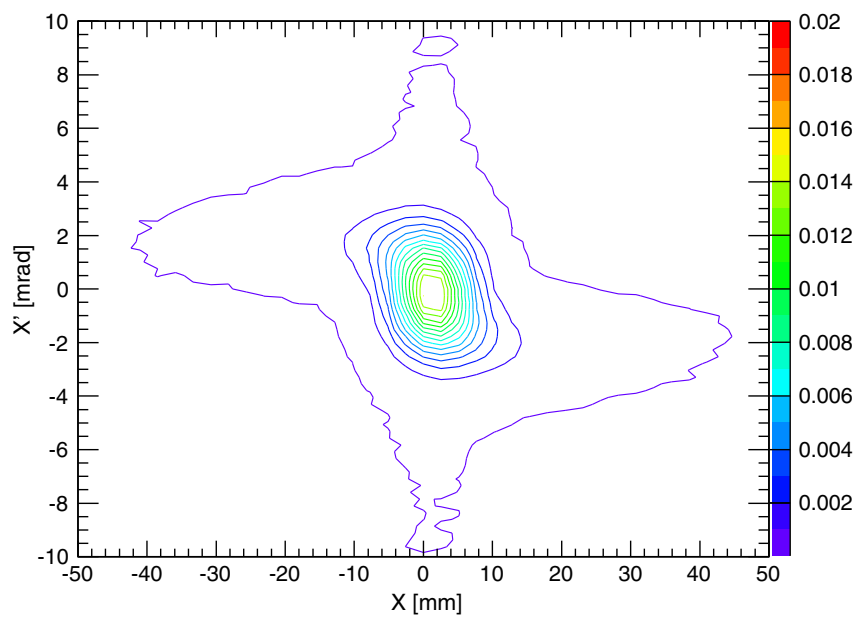

FIG. 20. Contour plot of transverse phase space distribution in the horizontal direction for beam scattered from the muon production target.

in Fig. 19. We observed that the octupole magnetic field generates an arm-shaped distribution in the phase space. The beam particles in the arm region play an important role in beam flattening at the mercury target. Figure 20 shows the beam distribution after the beam passes the carbon target. Beam divergence, which is plotted on the vertical axis of Fig. 20, is wide due to the scattering, so the beam distribution is nearly Gaussian. To maintain a flat distribution, this increase in beam divergence due to scattering should be suppressed. Therefore, the beam should be focused at the muon-production target to increase beam divergence there.

\section{CONCLUSION}

To reduce the peak current density of the beam at the target without using the rastering technique, beam transport with nonlinear optics based on octupole magnets was studied. To understand the focusing mechanism of nonlinear optics, a filament model was used in the phase distribution. It was found that previous works with different approaches showed similar results in terms of the octupolemagnet-strength $\left(K_{8}\right)$ requirements to obtain a flat beam shape on the target for reducing the peak current density there. Because the beam has infinite intensity at its edge according to the filament model, the spread of the angular distribution was taken into account. By selecting a phase advance such as $\cot \phi \sim \pm 3$, lower strengths of $K_{8}$ could be sustained such that the beam size can be kept smaller by defocusing using nonlinear optics downstream of the octupole.

A new generalized model is proposed for application with an octupole magnet regardless of whether the filament-model approximation is used. The transverse distribution at the arbitrary position can be characterized only by the normalized octupole strength of $K_{8}^{*}$ and the $\cot \phi$ of the 
phase advance from the octupole. With the generalized model, the best uniform shape can be found for certain values of $K_{8}^{*}$ and $\cot \phi$, which cannot be deduced from the filament model. From the beam-tracking calculation for various $K_{8}^{*}$ and $\cot \phi$, the best uniform shape can be obtained for $K_{8}^{*}$ and $\cot \phi$ of 3.5 and 5.8, respectively, for the beamwidth region in one sigma of the linear optics $\left(1 \sigma_{t}\right)$. However, to achieve the flat distribution, a larger $K_{8}^{*}$ incurring a significant beam loss downstream of the octupole having $\cot \phi \sim-0.5$. To achieve the two antagonistic requirements of beam flattening and minimization of beam loss, the beam loss was calculated for a large-aperture region for $2.5 \sigma_{t}$ and $6 \sigma_{t}$. It is found that a bell-shaped distribution with $K_{8}^{*} \sim 1$ and $\cot \phi \sim 3$ can satisfy requirements.

The beam profiles calculated by tracking with SAD were compared with the experimental results obtained using monitors placed $1.8 \mathrm{~m}$ upstream of the neutron-production target in J-PARC. The calculation results show good agreement with the experimental results for the 1-MW beam. Calculations considering beam scattering from the muon-production target indicate that the peak current density can be reduced by approximately $50 \%$ by introducing the present nonlinear beam optics without any significant increase in beam loss. To limit any increase in beam emittance due to scattering from the muonproduction target, the beam is focused on the muonproduction target to obtain a large beam divergence. Furthermore, we studied how magnetic misalignment affects the beam profile. With beam offset at the octupole magnet, the beam density at the target edge increases. The acceptable beam offset at the octupole magnet is approximately $1 \mathrm{~mm}$, which is a feasible value of precision considering present beam operation. The simulation results show that the alignment error of quadrupole magnets downstream of the octupole magnets does not influence the shape of the beam at the target. Octupole magnets have already been installed in the proton-transport line of the JSNS at J-PARC. The system described herein can reduce pitting erosion on the target vessel drastically. Note that the present study is the first attempt to install a nonlinear-optics system in a MW-class hadron accelerator. We proved that the transverse beam distribution with the nonlinear optics using an octupole magnet can be simplified by the present generalized model, which can be applied to other facilities such as the ADS and the IFMIF.

\section{ACKNOWLEDGMENTS}

The authors would like to convey their acknowledgments to Dr. Irie for a fruitful discussion about the design of the beam transport to the MLF and would like to show appreciation to Dr. Yuri and Dr. Méot for fruitful discussions on nonlinear optics and their encouragement of the present study. Also, the authors would like to show appreciation to the LINAC and RCS beam commissioning team for beam tuning and the target team for delivering $1 \mathrm{MW}$ beam from morning to midnight for this study.

\section{APPENDIX A: OPTIMIZATION OF MAGNETIC PHASE ADVANCE WITH THE FILAMENT MODEL}

By the filament-approximation model, although the beam divergence is ignored, the beam distribution can be simplified using a filament with Gaussian amplitude at the octupole. Using the filament model, the target distribution can be described by the simplified third-order equation given below:

$$
x_{t}=\lambda_{1} x_{1}+\lambda_{3} x_{1}^{3},
$$

where $\lambda_{1}$ and $\lambda_{3}$ are

$$
\begin{gathered}
\lambda_{1}=\sqrt{\beta_{t} / \beta_{1}} \cos \phi, \\
\lambda_{3}=-K_{8} \sqrt{\beta_{t} \beta_{1}} \sin \phi / 6,
\end{gathered}
$$

with $\beta_{t}$ being a beta function at the target, and $\phi$ being the phase advance between the octupole and the target. It should be mentioned that Eqs. (A1)-(A3) are similar to those reported by Méot $[29,30]$, but the present equations provide the beam position as well, which facilitates a straightforward understanding of the phenomena of nonlinear optics. Moreover, the previous works by Méot were expressed in terms of the angle of the beam at the octupole magnets. Since Eq. (A1) is of the third order, it may have three distinct real roots if the discriminant formula is positive. If the discriminant formula is negative, Eq. (A1) has a single real root. Moreover, Eq. (A1) has multiple roots at $x_{t s}$, which can be obtained from the discriminant formula in Eq. (A1),

$$
x_{t s}= \pm 2 / 3 \lambda_{1} \sqrt{-\lambda_{1} / 3 \lambda_{3}} .
$$

Given the differential of Eq. (A1) with $x_{1}$ as zero (i.e., $d x_{t} / d x_{1}=0$ ), the beam position at the octupole magnet $\left(\mathrm{x}_{1 s}\right)$ that yields multiple root points can be solved as

$$
x_{1 s}= \pm \sqrt{-\lambda_{1} / 3 \lambda_{3}} .
$$

From Eqs. (A4) and (A5), if $\lambda_{1} / 3 \lambda_{3}$ is negative, singular points exist. In Fig. 21, the typical distribution is shown with the filament model. The filament model is found to show the envelope of vertices for various beam emittances. Moreover, the phase-space distribution is shown to have concavity at $x_{t s}$, so the beam is folded and has a flat shape, which is fundamental for beam flattening.

If $\lambda_{1} / 3 \lambda_{3}$ is positive, the beam is not folded and expanded by nonlinear optics. As shown in Fig. 21, the beam is defocused by nonlinear optics, and the beamwidth increases drastically. In this case, the beam loss is larger 


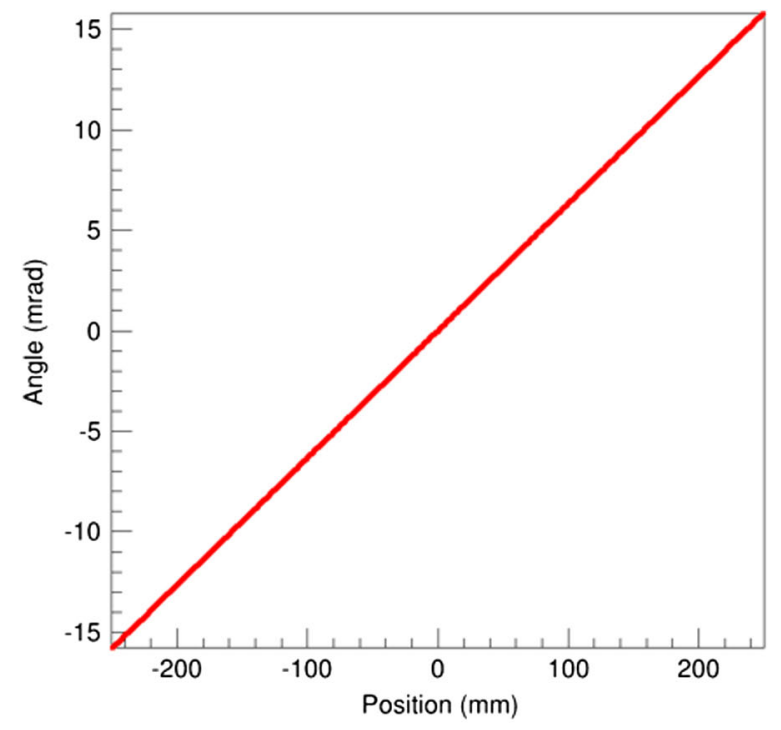

(a)

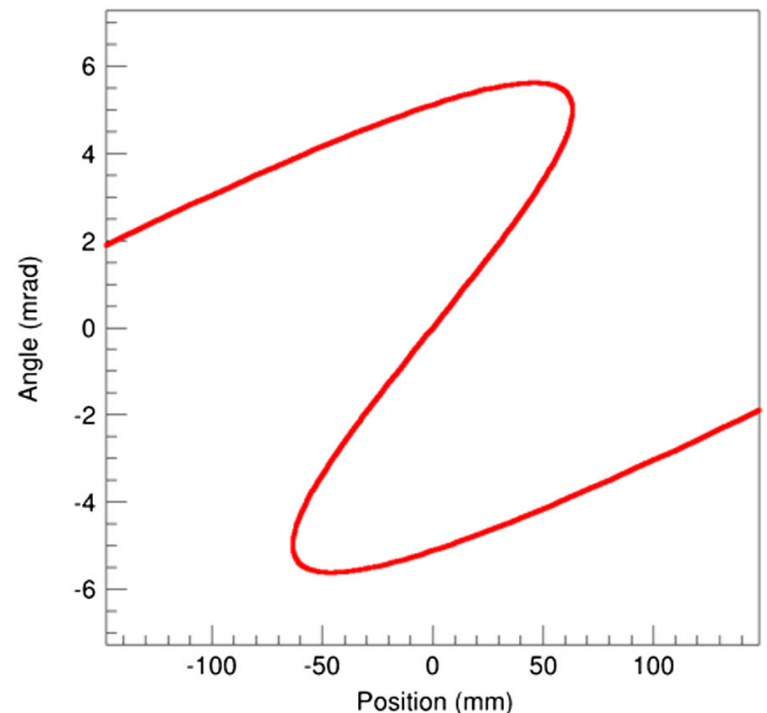

(b)

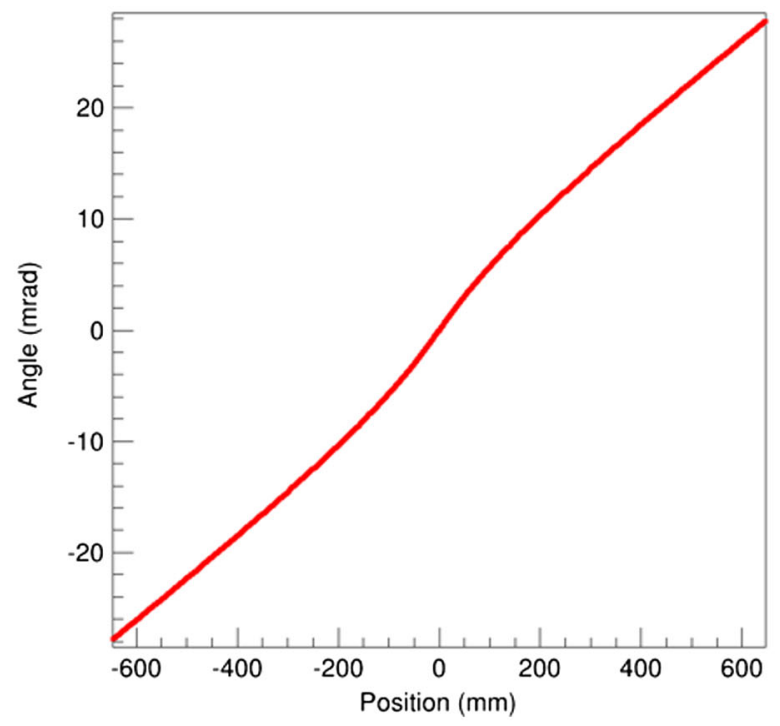

(c)

FIG. 21. Phase space distribution expressed by filament model for (a) linear optics, (b) nonlinear optics for focusing, and (c) nonlinear optics for defocusing.

than that in the linear-optics case. By solving Eq. (A1), the beam envelope $\left(x_{2}\right)$ at the downstream components such as the quadrupole magnet, which has beta function $\beta_{2}$ and phase advance $\varphi$ from the octupole, is derived from Eqs. (A1)-(A3) for the envelope of a beam with an arbitrary emittance. The beamwidth at the octupole magnet of $x_{1}$ corresponding to the transverse emittance $\epsilon(\pi \mathrm{mm} \cdot \mathrm{mrad})$ can be described as $\sqrt{\epsilon \beta_{1}}$ :

$$
x_{2}=\sqrt{\epsilon \beta_{2}}\left|\frac{\epsilon K_{8} \beta_{1}^{2} \sin \varphi}{6}-\cos \varphi\right| .
$$

From Eq. (A6), it is found that the maximum beamwidth obtained by defocusing using nonlinear optics is $\epsilon K_{8} \beta_{1}^{2} / 6$-times that achieved with linear optics for $\pi / 2+$ $n \pi(n=0,1,2, \ldots)$. It is also found that the maximum size is proportional to $K_{8}$. To suppress beam loss downstream of the octupole magnet, $K_{8}$ should be kept as small as possible. From the beam distribution at the octupole magnet, defined as $f\left(x_{1}\right)$, the beam distribution at the target, defined as $g\left(x_{t}\right)$, can be obtained by the conservation of integration particles of Eq. (A1) as follows:

$$
g\left(x_{t}\right)=f\left(x_{1}\right) d x_{1} / d x_{t}=f\left(x_{1}\right) /\left|\lambda_{1}+3 \lambda_{3} x_{1}^{2}\right| .
$$

When $\left|x_{t}\right|<\left|x_{t s}\right|, x_{1}$ for each $x_{t}$ has double real roots, then Eq. (A7) is described by the integration of each root. It should be noted that Eq. (A7) yields infinite amplitude for 
singular points because the denominators are zero, as will be discussed later.

As given by Eq. (A3), a small value of the integrated octupole magnetic field of $K_{8}$ should be chosen to suppress beam loss. Substituting Eqs. (A2) and (A3) into Eq. (A4), we can find $K_{8}$ to obtain a flat shape as follows:

$$
K_{8}=8 \beta_{t} \cos ^{3} \phi / 9 x_{t s}^{2} \beta_{1}^{2} \sin \phi .
$$

By choosing a certain $\mathrm{x}_{t s}$ to fit the target size, $K_{8}$ is determined from Eq. (A8). Here, two different approaches are considered to obtain an octupole magnetic field.

Méot and Aniel [29,30] studied nonlinear optics to obtain a uniform beam shape using octupole and dodecapole magnets. According to Ref. [29], for the singleoctupole-magnet case, the $x_{t s}$ required to obtain a flat distribution is empirically assumed to be $4 / 3 \sigma$, where $\sigma$ is beamwidth at the target for linear optics of $\sqrt{\epsilon_{\sigma} \beta_{t}}$ and $\epsilon_{\sigma}$ is one sigma of beam emittance. Therefore, the required magnetic field can be obtained as follows:

$$
K_{8}=\cos ^{3} \phi / 2 \epsilon_{\sigma} \beta_{1}^{2} \sin \phi=\cos ^{2} \phi / 2 \epsilon_{\sigma} \beta_{1}^{2} \tan \phi .
$$

Yuri et al. [23] studied nonlinear optics to obtain a flat distribution using infinite multipole magnets and the filament model, which is different from the approach adopted by Méot and Aniel. Using the Gaussian distribution in the initial condition of the transverse phase space, multipole magnetic fields up to infinity are determined to have completely flat shape at the target by means of the Taylor expansion of their Gaussian distributions,

$K_{2 n}=\frac{(n-2) !}{(n / 2-1) !\left(2 \epsilon_{\sigma} \beta\right)^{n / 2-1}} \cdot \frac{(-1)^{n / 2}}{\beta \tan \phi}(n=4,6,8, \ldots)$.

Equation (A10) can be used to obtain the required magnetic field in not only the octupole case, but also in other multipole magnet cases up to finitely high order. For the octupole magnet, the required field is expressed:

$$
K_{8}=\left(\epsilon_{\sigma} \beta_{1}^{2} \tan \phi\right)^{-1} \text {. }
$$

It should be noted that complete flatness cannot be achieved using only octupole magnets whose peaks appear at the side of the beam on the target. For this, higher-order magnets of up to 20 poles may be necessary. In this study, we use only an octupole magnet for simplicity. It has been shown that the octupole field adjusted by Eq. (A11) has a side peak owing to tracking of the beam, and half of the strength given by Eq. (A11) is adequate for achieving a flatness within 20\%, as shown in Fig. 2 of Ref. [23]. For cases with lower energy and lower power, placing a collimator at the side edge can easily remove the side peak, but this method is quite difficult to execute in a high-power beam facility such as JSNS. In Ref. [23], the half-width-beam flat region $(r)$ at the target was expressed as follows:

$$
r=\sqrt{\pi / 2} \sqrt{\epsilon_{\sigma} \beta_{t}}|\cos \phi|
$$

If $r$ is chosen to be the singularity ( $\left.\mathrm{x}_{t s}\right)$ in Eq. (11), $K_{8}$ is obtained as follows:

$$
K_{8}=16 / 9 \pi \epsilon_{\sigma} \beta_{1}^{2} \tan \phi \simeq 0.566 / \epsilon_{\sigma} \beta_{1}^{2} \tan \phi,
$$

which is similar to Eq. (A9) as obtained by the approach of Méot, except for the term $\cos ^{2} \phi$. Equation (A13) also shows consistency with the result of Ref. [23] that flatness is within 20\%. From Eqs. (A9) and (A13), it is recognized that $\beta_{1}$ should be larger, but a larger value leads to increased beam loss at the octupole magnet. It should be noted that the magnet's polarity is also given by Eqs. (A9) and (A13). Here, for the sake of simplicity, a positive case is discussed.

\section{APPENDIX B: OPTIMIZATION OF PHASE ADVANCE WITH THE FILAMENT MODEL}

Owing to the use of nonlinear optics, the beam downstream of the octupole magnet is expanded drastically. To minimize beam loss using the filament model, here, we discuss the beam size under the condition needed to avoid beam expansion using nonlinear optics and an octupole magnet. By omitting the term in Eq. (A6) corresponding to the phase advance between the octupole and any arbitrary downstream position, the maximum beam size achievable using nonlinear optics is proportional to $\epsilon \mathrm{K}_{8} \beta_{1}^{2}$ for linear optics of size $\sqrt{\epsilon \beta_{2}}$. Equations (A9) and (A13) show that the $\epsilon_{\sigma} K_{8} \beta_{1}^{2}$ term is proportional to $\cot \phi$. Because $\epsilon$ and $\epsilon_{\sigma}$ are determined by the upstream accelerator condition, the value is invariant along the direction of beam transport. Thus, to obtain a flat beam distribution with lower beam status, the values of $K_{8}$ and $\beta_{1}$ should be set lower by choosing the appropriate phase advance.

To understand the dependence of phase advance upon the beam profile at the target, the phase-space distribution and beam profile at the target were calculated by tracking, as shown in Figs. 22 and 23, respectively, for various $\cot \phi$ using $K_{8}$ determined via Eq. (A13). For the sake of convention, a normalized Gaussian distribution was used in the phase space as the origin of particles. It is found that the phase-space distribution for $\cot \phi \sim 1$ is similar to that for linear optics, so the beam profile becomes Gaussian at the target. In this case, the nonlinear optics does not influence the beam shape at the target, which leads to a small beamwidth for folding with the nonlinear optics, as given by Eq. (B1). As shown in Fig. 22, the phase-space distribution deforms with increasing $\cot \phi$. Owing to this deformation, the beam shape flattens, as shown by the black line in Fig. 23, where beam intensity is normalized against the result obtained with the linear-optics calculation. For the lower case of $\cot \phi \sim 1$ 


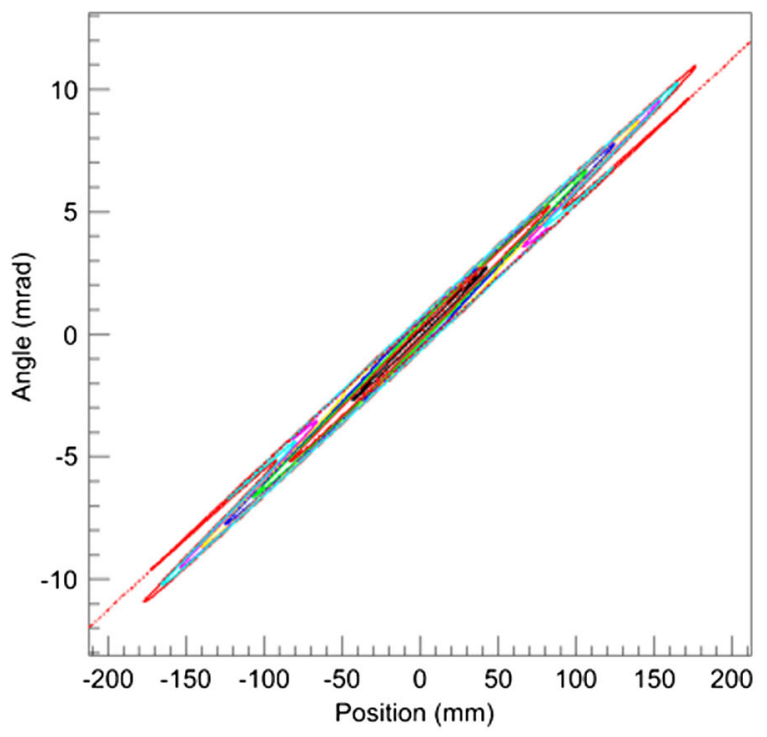

(a)

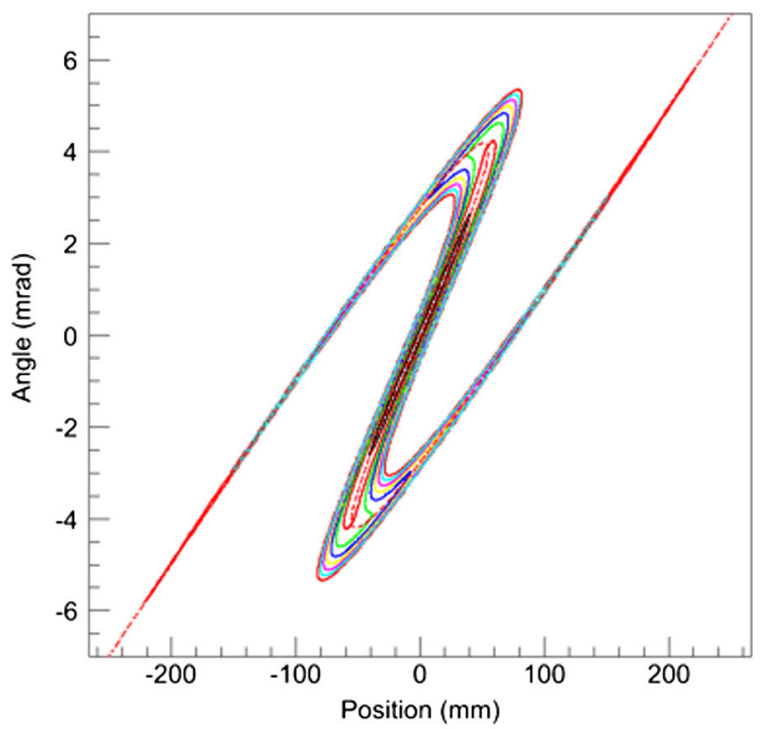

(c)

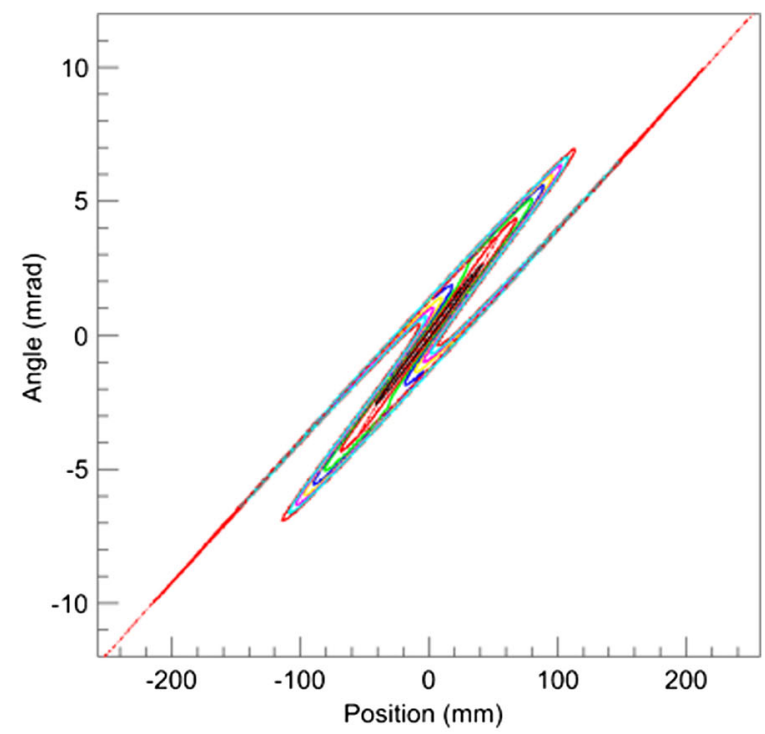

(b)

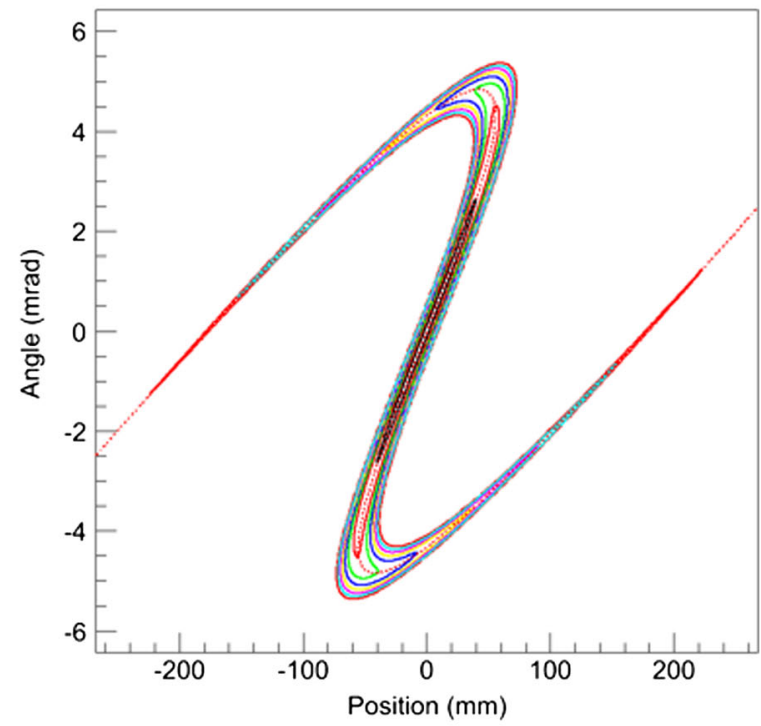

(d)

FIG. 22. Transverse phase space distribution at target obtained by tracking with octupole strength $\left(K_{8}\right)$ obtained using Eq. (A13) for various phase advance cases of $\cot \phi 1,3,5$, and 7 shown in panels (a)-(d), respectively.

shown in Fig. 23(a), defocusing due to the nonlinear optics is not significant because the strength requirement of $K_{8}$ is low; however, the beam shape is close to that obtained by linear optics. The beam has the flat distribution shown in Figs. 23(b)-23(d) as $\cot \phi$ increases. It is found that considerable flatness can be obtained with the phase advance of $\cot \phi \sim 3$, as shown in Fig. 23(b). Therefore, this value of phase advance should be selected. The peak intensity in the nonlinear case is shown to be the same as that for linear optics, except for the case of $\cot \phi \sim 7$, in which case the peak is slightly higher than that using linear optics. A comparison between Figs. 6(b) and 6(c) shows that the flat region is enlarged as $\cot \phi$ increases. For $\cot \phi$ larger than 11 , a side-edge peak appears in Fig. 23(d). In this case, the strength of $K_{8}$ is excessive for the flattening required for the present purpose, and it can increase beam loss due to nonlinear beam expansion.

To analytically understand the dependence of the beam profile upon the phase advance, we consider the beam distribution obtained by the filament model. The beam distribution obtained by the filament model using Eq. (A7) is shown as a green line in Fig. 23, suggesting good agreement with the tracking result for the case of $\cot \phi$ larger than 3 , except at the side edge. The side edge appears at $1.2 \sigma$, which is close to the empirical result obtained by Méot [29]. While the beam profile can be obtained with the filament model 


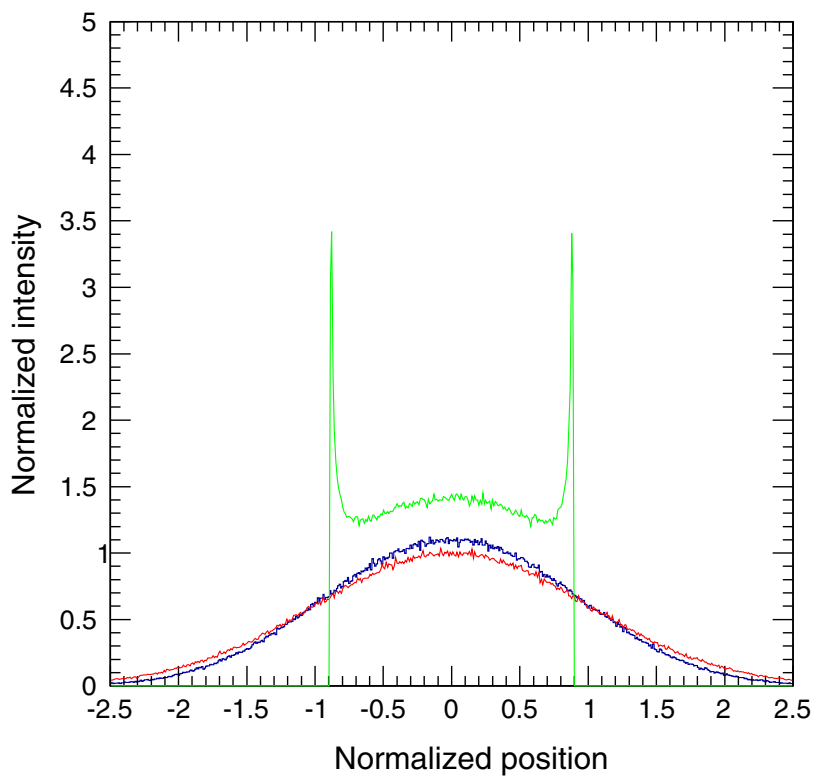

(a)

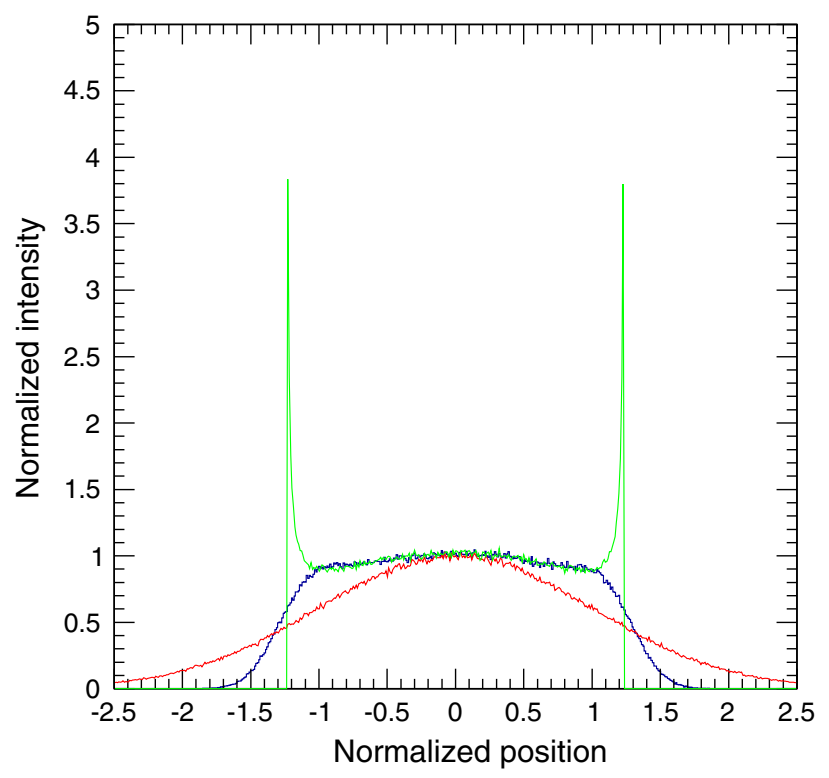

(c)

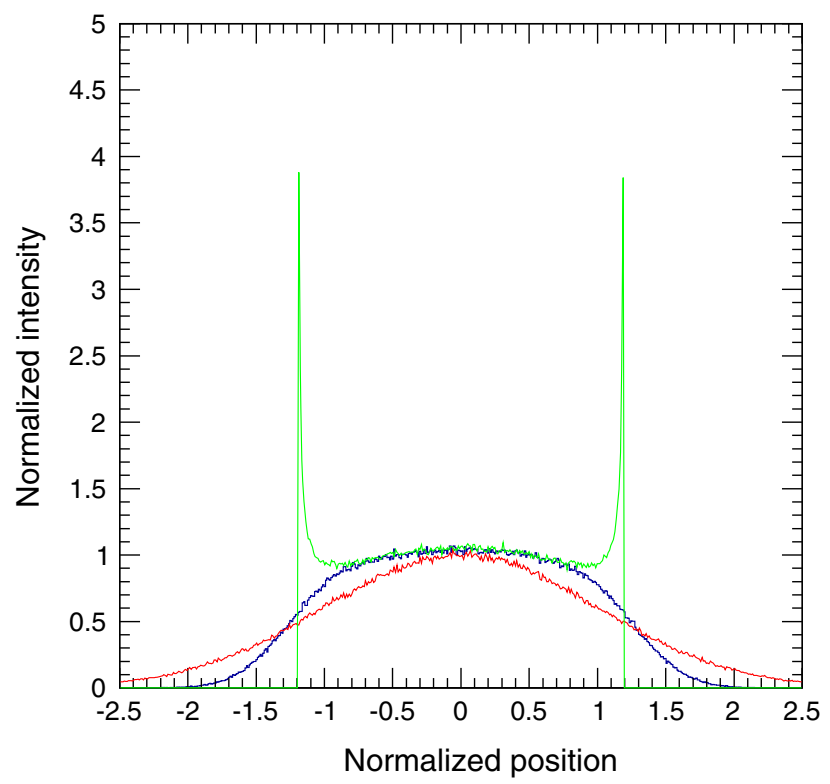

(b)

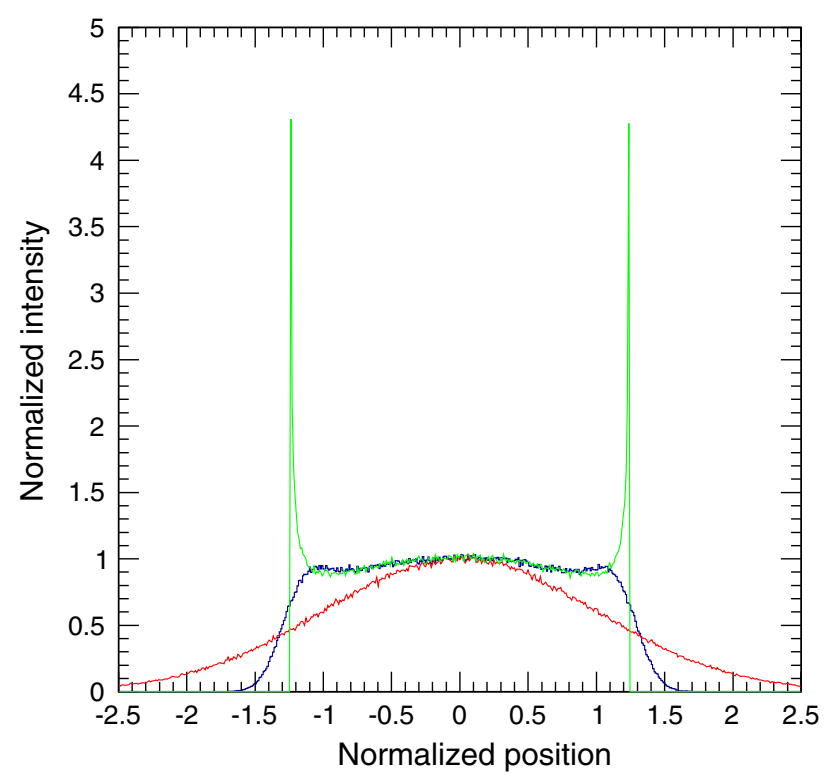

(d)

FIG. 23. Transverse beam distribution obtained by tracking for various phase advance (black lines) of $\cot \phi=1,3,5$, and 7 shown in panels (a)-(d), respectively, with Gaussian-normalized phase space distribution. The red line shows the profile obtained with linear optics, and the green line shows the result obtained using the filament model.

using Eq. (10), the amplitude at the side edge becomes infinite due to singularity. This fact is caused by the finite intensity converging at the singularity point, where the filament becomes perpendicular to the position in the phase space shown in Fig. 21 and the filament width becomes zero. Although Eq. (A7) gives an infinite value, the intensity for the filament model shown in Fig. 23 goes to infinity because of the position resolution in the numerical calculation.

While the intensity at the side edge of the beam determines beam flatness, the filament model cannot give an accurate value of intensity because it ignores the spread of the angular distribution. To analytically discuss the effect of phase advance on beam shape, we introduce an angular distribution into the filament model. The beam position at $x_{1 s}$, which is the position of singularity on the target, is expressed as

$$
x_{1 s}^{2}=-\lambda_{1} / 3 \lambda_{3}=2 / K_{8} \beta_{1} \tan \phi,
$$

where $K_{8}$ is from Eq. (A13) and $x_{1 s}$ is expressed as 


$$
x_{1 s}^{2}=9 \pi \epsilon_{\sigma} \beta_{1} / 8 .
$$

Here, without approximation and with the angular distribution at the octupole, the beam position at the singularity of the target is expressed by Eqs. (3), (A2), and (A3):

$x_{t}=\lambda_{1} x_{1}+\lambda_{3} x_{1}^{3}+\sqrt{\beta_{t} / \beta_{1}} \sin \phi\left(\alpha_{1} x_{1}+\beta_{1} p x_{1}\right)$,

where $p x_{1 s}$ is the angle at the octupole having singularity (i.e., $p x_{1 s}=-\alpha_{1} / \beta_{1} \cdot x_{1 s}$ ), and there is a small deviation around the singularity as follows:

$$
\Delta t / \alpha_{1} \equiv x_{1}-x_{1 s}, \quad \Delta t / \beta_{1} \equiv p x_{1}-p x_{1 s} .
$$

These small differences in singularity at the octupole cause the following variation in the position of singularity:

$$
\begin{aligned}
x_{t}+\Delta x_{t}= & \lambda_{1}\left(x_{1 s}+\Delta t / \alpha_{1}\right)+\lambda_{3}\left(x_{1 s}+\Delta t / \alpha_{1}\right)^{3} \\
& +\sqrt{\beta_{t} / \beta_{1}} \sin \phi\left[\alpha_{1}\left(x_{1 s}+\Delta t / \alpha_{1}\right)\right. \\
& \left.+\beta_{1}\left(p x_{1 s}+\Delta t / \beta_{1}\right)\right] .
\end{aligned}
$$

By subtracting Eq. (B5) from Eq. (B3) and ignoring highorder $\Delta t$, we have

$\Delta x_{t}=\left(\Delta t / \alpha_{1}\right)\left(\lambda_{1}+3 \lambda_{3} x_{1 s}^{2}\right)+\sqrt{\beta_{t} / \beta_{1}} \sin \phi(2 \Delta t)$.

Because the first term is zero at the singularity position,

$$
\Delta x_{t}=2 \sqrt{\beta_{t} / \beta_{1}} \sin \phi \Delta t .
$$

Therefore, the intensity at the singularity can be obtained as follows:

$$
g\left(x_{t}\right) d x_{t}=f\left(x_{1 s}\right) d t .
$$

From Eq. (B7), the intensity of the singularity can finally be obtained as

$$
\begin{aligned}
g\left(x_{t s}\right) & =f\left(x_{1 s}\right) d t / d x_{t}=\frac{f\left(x_{1 s}\right)}{2 \sqrt{\beta_{t} / \beta_{1}} \sin \phi} \\
& =\frac{1}{2 \sqrt{2 \pi \beta_{t} \epsilon_{\sigma}} \sin \phi} \exp \left(-\frac{x_{1 s}^{2}}{2 \epsilon_{\sigma} \beta_{1}}\right) .
\end{aligned}
$$

From $x_{1 s}$ obtained using Eq. (B2), the intensity at the singularity can be obtained as

$$
g\left(x_{t s}\right)=\frac{\exp (-9 \pi / 16)}{2 \sqrt{2 \pi \beta_{t} \epsilon_{\sigma}} \sin \phi} .
$$

Here, we consider the relative intensity at the singularity point on the target center. Because Eq. (A1) has two real roots at the center for the nonlinear-focus case, the intensity center is expressed by integrating the intensity of those roots. For the flat shape case at the target, because the intensity given by the tail of the beam at the octupole magnet (which appears at the center of the target) is much smaller than that given by the center of the beam at the octupole, the intensity at the center can be expressed as follows:

$$
g(0)=\left(2 \pi \epsilon_{\sigma} \beta_{t}\right)^{-0.5} .
$$

From Eqs. (B10) and (B11), the ratio of intensity at the singularity to that at the center $(F)$ can be expressed as

$$
F \equiv g\left(x_{t s}\right) / g(0)=\exp (-9 \pi / 16) / 2 \sin \phi .
$$

The relationship between $F$ and $\phi$ can be expressed as

$$
\phi=\sin ^{-1}[\exp (-9 \pi / 16) / 2 F] .
$$

As shown in Eq. (B13), $F$ can be expressed as a general parameter without dependence on the Twiss parameter for both the target and the octupole magnet. To confirm this result, we performed some calculations with beam tracking for various values of phase advance, $\phi$. In Table III, the dependence of $F$ on $\phi$ is shown. Note that the case of $F=1 / e$ has the same value of $\phi=13.4^{\circ}$ as in the previous work [55] for the Chinese Spallation Neutron Source, which is, however, discussed for limited optics with having a waist (i.e., $\alpha=0$ ) at the octupole magnet. The relative beam shape is given by $K_{8}$ and the phase advance, so that beam shape can be described irrespective of the Twiss parameter of $\alpha$ at the octupole and the target creating a degree of freedom for beam tuning with nonlinear optics.

Table III also shows the beam loss for various $F$. The beam loss was calculated for the aperture having $\pm 3 \sigma$ width of the linear optics at the position where the maximum beam loss occurs $\cot \varphi=-0.5$ as shown in Fig. 7. As $F$ increases, the beam loss increases because the required $K_{8}^{*}$ becomes larger.

TABLE III. Relationship between relative intensity at edge to center $(F)$, phase advance $\phi$ of obtained using Eq. (B13), $K_{8}^{*}$ obtained using Eqs. (A13) and (16), and the maximum beam loss for the aperture having $\pm 3 \sigma$ of the linear optics at $\cot \varphi=-0.5$.

\begin{tabular}{lcrrr}
\hline \hline$F$ & $\phi[\mathrm{deg}]$ & $\cot \phi$ & $K_{8}^{*}$ & Beam loss \\
\hline 1 & 4.9 & 11.6 & 6.6 & $1.9 \times 10^{-1}$ \\
0.7 & 7.0 & 8.1 & 4.6 & $1.5 \times 10^{-1}$ \\
0.5 & 9.8 & 5.8 & 3.3 & $1.1 \times 10^{-1}$ \\
$0.37\left(=e^{-1}\right)$ & 13.4 & 4.2 & 2.4 & $8.2 \times 10^{-2}$ \\
\hline \hline
\end{tabular}




\section{APPENDIX C: BEAM LOSS WITH FILAMENT-APPROXIMATION MODEL}

In a high-intensity facility, control over beam loss is crucial. Owing to the defocusing caused by nonlinear optics, beam size at the tail, which is described by a certain emittance $\epsilon$ in the phase space, can be expressed in terms of Eq. (A6) and the $K_{8}$ value obtained using Eq. (A13),

$$
\begin{aligned}
x_{2} & =\sqrt{\epsilon \beta_{2}}\left|\frac{\epsilon K_{8} \beta_{1}^{2} \sin \phi}{6}-\cos \varphi\right| \\
& =\sqrt{\epsilon \beta_{2}}\left|\frac{8 \epsilon \sin \varphi}{27 \pi \epsilon_{\sigma} \tan \phi}-\cos \varphi\right| .
\end{aligned}
$$

Thus, the maximum size of the beam envelope is given by the following equation:

$$
x_{2} \leq \frac{8}{27 \pi} \sqrt{\epsilon \beta_{2}}\left(\epsilon / \epsilon_{\sigma}\right) \cot \phi .
$$

As shown in Eq. (C2), beam size is greater than that in the linear-optics case, and this increase is proportional to $\cot \phi$ and the relative amplitude of emittance is proportional to rms $\left(\epsilon_{\sigma}\right)$. Again, to achieve lower beam loss, a lower value of $\cot \phi$ should be selected. On the contrary, for the case of larger $\cot \phi \sim 11$, a relatively flat distribution can be obtained. As a matter of fact, a tradeoff is required to achieve the contradictory demands of low beam loss and flat shape. As shown in Fig. 23(b), the bell-shaped beam corresponding to $\cot \phi \sim 3$ and a phase advance of $18^{\circ}$ can be thought of as a solution for simultaneously achieving the requirements of beam flattening and reduced beam loss. In general, the beam size increases at the focus and defocus of the quadrupole magnets in the horizontal and vertical planes, respectively. In the defocus phase with nonlinear optics, beam expansion is proportional to emittance and $\cot \phi$. From Eq. (C1), it can be seen that beam expansion appears when $\varphi=n \pi+\pi / 2$ (for $n=0,1,2, \ldots$ ). Because the beam expansion in the linear optics appears at the focusing and defocusing magnets' midplane in the horizontal and vertical directions, respectively, beam expansion can be minimized by choosing suitable $\phi$ values at each quadrupole magnet between the octupole and the quadrupole magnets.

[1] K. Tsujimoto, H. Oigawa, N. Ouchi, K. Kikuchi, Y. Kurata, M. Mizumoto, T. Sasa, S. Saito, K. Nishihara, M. Umen et al., Research and development program on accelerator driven subcritical system in JAEA, J. Nucl. Sci. Technol. 44, 483 (2007).

[2] H. A. Abderrahim, P. Baeten, D. D. Bruyn, J. Heyse, P. Schuurmans, and J. Wagemans, MYRRHA, a Multipurpose
hYbrid research reactor for high-end applications, Nucl. Phys. News 20, 24 (2010).

[3] R. Schmidt, R. Assmann, E. Carlier, B. Dehning, R. Denz, B. Goddard, E. B. Holzer, V. Kain, B. Puccio, B. Todd et al., Protection of the CERN Large Hadron Collider, New J. Phys. 8, 290 (2006).

[4] R. B. Appleby, B. Goddard, A. Gomez-Alonso, V. Kain, T. Kramer, D. Macina, R. Schmidt, and J. Wenninger, Beamrelated machine protection for the CERN Large Hadron Collider experiments, Phys. Rev. ST Accel. Beams 13, 061002 (2010).

[5] N. A. Tahir, J. B. Sancho, A. Shutov, R. Schmidt, and A. R. Piriz, Impact of high energy high intensity proton beams on targets: Case studies for Super Proton Synchrotron and Large Hadron Collider, Phys. Rev. ST Accel. Beams 15, 051003 (2012).

[6] Home page of RaDIATE collaboration, https://radiate .fnal.gov.

[7] R. Garoby, A. Vergara, H. Danared, I. Alonso, E. Bargallo, B. Cheymol, C. Darve, M. Eshraqi, H. Hassanzadegan, A. Jansson et al., The European spallation source design, Phys. Scr. 93, 014001 (2017).

[8] JAERI-Tech 99-56, JHF-99-3, and KEK Report, Japan Atomic Energy Research Institute, 1999.

[9] Y. Ikeda, J-PARC status update, Nucl. Instrum. Methods Phys. Res., Sect. A 600, 1 (2009).

[10] Y. Miyake, K. Shimomura, N. Kawamura, P. Strasser, S. Makimura, A. Koda, H. Fujimori, K. Nakahara, R. Kadono, M. Kato et al., Birth of an intense pulsed muon source, J-PARC MUSE, Physica (Amsterdam) 404B, 957 (2009).

[11] J. Haines, T. McManamy, T. Gabriel, R. Battle, K. Chipley, J. Crabtree, L. Jacobs, D. Lousteau, M. Rennich, and B. Riemer, Spallation neutron source target station design, development, and commissioning, Nucl. Instrum. Methods Phys. Res., Sect. A 764, 94 (2014).

[12] S. Meigo, F. Noda, S. Ishikura, M. Futakawa, S. Sakamoto, and Y. Ikeda, Evaluation of the $3-\mathrm{GeV}$ proton beam profile at the spallation target of the JSNS, Nucl. Instrum. Methods Phys. Res., Sect. A 562, 569 (2006).

[13] S. Sakamoto, S. Meigo, H. Fujimori, M. Harada, C. Konno, Y. Kasugai, T. Kai, Y. Miyake, and Y. Ikeda, Advanced design of high-intensity beam transport line in J-PARC, Nucl. Instrum. Methods Phys. Res., Sect. A 562, 638 (2006).

[14] S. Meigo, M. Ohi, T. Kai, T. Ono, K. Ikezaki, T. Haraguchi, H. Fujimori, and S. Sakamoto, Beam commissioning for neutron and muon facility at J-PARC, Nucl. Instrum. Methods Phys. Res., Sect. A 600, 41 (2009).

[15] D. McClintock, B. Riemer, P. Ferguson, A. Carroll, and M. Dayton, Initial observations of cavitation-induced erosion of liquid metal spallation target vessels at the Spallation Neutron Source, J. Nucl. Mater. 431, 147 (2012).

[16] T. Naoe, H. Kogawa, T. Wakui, K. Haga, M. Teshigawara, H. Kinoshita, H. Takada, and M. Futakawa, Cavitation damage prediction for the JSNS mercury target vessel, J. Nucl. Mater. 468, 313 (2016).

[17] M. Futakawa, T. Naoe, H. Kogawa, C.-C. Tsai, and Y. Ikeda, Pitting damage formation up to over 10 million cycles, J. Nucl. Sci. Technol. 40, 895 (2003). 
[18] M. Futakawa, Material issues relating to high power spallation neutron sources, IOP Conf. Ser. 74, 012001 (2015).

[19] M. Futakawa, T. Naoe, H. Kogawa, M. Teshigawara, and Y. Ikeda, Effects of pitting damage on fatigue limit and lifetime in mercury target, J. Nucl. Mater. 356, 168 (2006).

[20] T. Naoe, M. Teshigawara, T. Wakui, H. Kinoshita, H. Kogawa, K. Haga, and M. Futakawa, Damage inspection of the first mercury target vessel of JSNS, J. Nucl. Mater. 450, 123 (2014).

[21] S. Meigo, M. Ohi, S. Sakamoto, M. Futakawa, and H. Fujimori, in Proceedings of the 1st International Particle Accelerator Conference, Kyoto, Japan (ICR, Kyoto, 2010), p. MOPEB066.

[22] T. Naoe, M. Futakawa, T. Shoubu, T. Wakui, H. Kogawa, H. Takeuchi, and M. Kawai, Mitigation technologies for damage induced by pressure waves in high-power mercury spallation neutron sources (I) - material surface improvement-, J. Nucl. Sci. Technol. 45, 698 (2008); https://www.tandfonline.com/doi/abs/10.1080/18811248 .2008.9711469.

[23] Y. Yuri, N. Miyawaki, T. Kamiya, W. Yokota, K. Arakawa, and M. Fukuda, Uniformization of the transverse beam profile by means of nonlinear focusing method, Phys. Rev. ST Accel. Beams 10, 104001 (2007).

[24] P.F. Meads, A nonlinear lens system to smooth the intensity distribution of a gaussian beam, IEEE Trans. Nucl. Sci. 30, 2838 (1983).

[25] E. Kashy and B. Sherrill, A method for the uniform charged particle irradiation of large targets, Nucl. Instrum. Methods Phys. Res., Sect. B 26, 610 (1987).

[26] B. Sherrill, J. Bailey, E. Kashy, and C. Leakeas, Use of multipole magnetic fields for making uniform irradiations, Nucl. Instrum. Methods Phys. Res., Sect. B 40-41, 1004 (1989).

[27] B. Blind, Production of uniform and well-confined beams by nonlinear optics, Nucl. Instrum. Methods Phys. Res., Sect. B 56-57, 1099 (1991).

[28] Y. Batygin, Beam intensity redistribution in a nonlinear optics channel, Nucl. Instrum. Methods Phys. Res., Sect. B 79, 770 (1993).

[29] F. Méot and T. Aniel, Principles of the non-linear tuning of beam expanders, Nucl. Instrum. Methods Phys. Res., Sect. A 379, 196 (1996).

[30] F. Méot and T. Aniel, Calculation of nonlinear envelopes in beam expanders, Phys. Rev. ST Accel. Beams 3, 103501 (2000).

[31] N. Tsoupas, R. Lankshear, C. L. Snead, J. T. E. Ward, M. Zucker, and H. A. Enge, in Proceedings of the 1991 IEEE Particle Accelerator Conference (PAC 1991), edited by L. Lizama and J. Chew (IEEE, Piscataway, NJ, 1991).

[32] N. Tsoupas, M. S. Zucker, T. E. Ward, and C. L. Snead, Uniform particle beam distributions produced by octupole focusing, Nucl. Sci. Eng. 126, 71 (1997).

[33] S. Richter and W. Barth, in Proceedings of the 8th European Particle Accelerator Conference, Paris, 2002 (EPS-IGA and CERN, Geneva, 2002), p. 1181.

[34] M. Martone, Report No. ENEA-RT-ERG-FUS-96-11, Italy, 1997, http://inis.iaea.org/search/search.aspx?orig_q= $\mathrm{RN}: 28050061$.
[35] J. Tang, H. Li, S. An, and R. Maier, Distribution transformation by using step-like nonlinear magnets, Nucl. Instrum. Methods Phys. Res., Sect. A 532, 538 (2004).

[36] C. Ronsivalle, C. Cianfarani, G. Messina, G. L. Orlandi, L. Picardi, E. Cisbani, and S. Frullani, in Proceedings of the 10th European Particle Accelerator Conference, Edinburgh, Scotland, 2006, edited by C. Prior (EPS-AG, Edinburgh, Scotland, 2006), pp. 2316-2318.

[37] N. Tsoupas, L. Ahrens, S. Bellavia, R. Bonati, K. A. Brown, I.-H. Chiang, C. J. Gardner, D. Gassner, S. Jao, W. W. Mackay et al., Uniform beam distributions at the target of the NASA Space Radiation Laboratory's beam line, Phys. Rev. ST Accel. Beams 10, 024701 (2007).

[38] A. Bogdanov, V. Anferov, M. Ball, D. V. Baxter, V. P. Derenchuk, A. V. Klyachko, T. Rinckel, and K. Solberg, in Proceedings of the 22nd Particle Accelerator Conference, PAC-2007, Albuquerque, NM (IEEE, New York, 2007), p. 1748 .

[39] R. Brinkmann and P. Raimondi, in Proceedings of the Particle Accelerator Conference, Chicago, IL, 2001 (IEEE, New York, 2001), Vol. 5, pp. 3828-3830.

[40] R. E. Shafer, Comment on "Uniformization of the transverse beam profile by means of nonlinear focusing method", Phys. Rev. ST Accel. Beams 11, 039001 (2008).

[41] Y. Yuri, N. Miyawaki, T. Kamiya, W. Yokota, K. Arakawa, and M. Fukuda, Reply to "Comment on 'Uniformization of the transverse beam profile by means of nonlinear focusing method",, Phys. Rev. ST Accel. Beams 11, 039002 (2008).

[42] H. Wiedemann, Particle Accelerator Physics (Springer International Publishing, AG Switerland, 2019), https:// doi.org/10.1007/978-3-319-18317-6.

[43] H. Hotchi, M. Kinsho, K. Hasegawa, N. Hayashi, Y. Hikichi, S. Hiroki, J. Kamiya, K. Kanazawa, M. Kawase, F. Noda et al., Beam commissioning of the $3-\mathrm{GeV}$ rapid cycling synchrotron of the Japan Proton Accelerator Research Complex, Phys. Rev. ST Accel. Beams 12, 040402 (2009).

[44] H. Hotchi, H. Harada, N. Hayashi, S. Kato, M. Kinsho, K. Okabe, P. K. Saha, Y. Shobuda, F. Tamura, N. Tani et al., Achievement of a low-loss 1-MW beam operation in the $3-\mathrm{GeV}$ rapid cycling synchrotron of the Japan Proton Accelerator Research Complex, Phys. Rev. Accel. Beams 20, 060402 (2017).

[45] H. Hotchi, H. Harada, P. Saha, Y. Shoubuda, F. Tamura, Y. Yamamoto, M. Yamamot, M. Yoshimoto, Y. Irie, T. Koseki et al., in Proceedings of the 3rd International Particle Accelerator Conference, New Orleans, LA, 2012 (IEEE, Piscataway, NJ, 2012), p. THPPP080.

[46] S. Meigo, M. Ooi, M. Harada, H. Kinoshita, and A. Akutsu, Radiation damage and lifetime estimation of the proton beam window at the Japan Spallation Neutron Source, J. Nucl. Mater. 450, 141 (2014).

[47] H. Fujimori, N. Kawamura, S. Meigo, P. Strasser, K. Nakahara, and Y. Miyake, Radiation resistant magnets for the J-PARC muon facility, Nucl. Instrum. Methods Phys. Res., Sect. A 600, 170 (2009).

[48] M. Yoshimoto, H. Harada, K. Okabe, and M. Kinsho, in Proceedings of the 54th ICFA Advanced Beam Dynamics Workshop on High-Intensity, High Brightness and High 
Power Hadron Beams (HB2014): East Lansing, Michigan, 2014 (ICFA FRIB, 2014), p. MOPAB44.

[49] K. Brown and C. Iselin, CERN Report No. CERN 74-2, 1974.

[50] U. Rohrer, PSI Graphic Turtle framework based on a CERN-SLAC-FERMILAB version, 2007).

[51] Home page of SAD, http://acc-physics.kek.jp/SAD.

[52] H. Hotchi, H. Harada, N. Hayashi, M. Kinsho, P. K. Saha, Y. Shobuda, F. Tamura, K. Yamamoto, M. Yamamoto, M. Yoshimoto et al., Beam loss reduction by injection painting in the 3-GeV rapid cycling synchrotron of the Japan Proton Accelerator Research Complex, Phys. Rev. ST Accel. Beams 15, 040402 (2012).
[53] M. J. Shirakata, Y. Fujii, T. Ishii, Y. Shirakabe, H. Harada, S. Harjo, T. Iwahashi, S. Meigo, T. Morishita, and N. Tani, in Proceedings of the 2nd International Particle Accelerator Conference, San Sebastián, Spain (EPS-AG, Spain, 2011), p. TUPS057.

[54] M. Harada, S. Meigo, M. Ito, E. Dantsuji, K. Takagiwa, H. Takada, F. Maekawa, M. Futakawa, M. Nakamura, Y. Miyake et al., Settlement of materials and life science experimental facility at J-PARC, Nucl. Instrum. Methods Phys. Res., Sect. A 600, 87 (2009).

[55] Y. Y. Guo and G. Xu, Design of transport lines for uniforming beam distribution with octupoles, Phys. Rev. ST Accel. Beams 16, 023501 (2013). 